\title{
Scaling Limits for the Gibbs States \\ on Distance-Regular Graphs with Classical Parameters
}

\author{
Masoumeh KOOHESTANI ${ }^{\mathrm{a}}$, Nobuaki OBATA ${ }^{\mathrm{b}}$ and Hajime TANAKA ${ }^{\mathrm{b}}$
}

a) Department of Mathematics, K.N. Toosi University of Technology, Tehran 16765-3381, Iran E-mail:m.kuhestani@email.kntu.ac.ir

b) Research Center for Pure and Applied Mathematics, Graduate School of Information Sciences, Tohoku University, Sendai 980-8579, Japan

E-mail: obata@tohoku.ac.jp, htanaka@tohoku.ac.jp

URL: https://www.math.is.tohoku.ac.jp/ obata/, https://www.math.is.tohoku.ac.jp/ htanaka/

Received July 19, 2021, in final form November 22, 2021; Published online November 26, 2021 https://doi.org/10.3842/SIGMA.2021.104

\begin{abstract}
We determine the possible scaling limits in the quantum central limit theorem with respect to the Gibbs state, for a growing distance-regular graph that has so-called classical parameters with base unequal to one. We also describe explicitly the corresponding weak limits of the normalized spectral distribution of the adjacency matrix. We demonstrate our results with the known infinite families of distance-regular graphs having classical parameters and with unbounded diameter.
\end{abstract}

Key words: quantum probability; quantum central limit theorem; distance-regular graph; Gibbs state; classical parameters

2020 Mathematics Subject Classification: 46L53; 60F05; 05E30

\section{Introduction}

Quantum probability theory is a non-commutative extension of classical probability theory; see, e.g., $[1,12,27,28,31,42]$. This paper is a contribution to the spectral analysis of growing graphs from the viewpoint of this theory. The adjacency matrix of a graph is regarded as a random variable in this context. (Formal definitions will be given in Section 2.) As in many previous works on this topic, our focus will be on central limit theorems (CLTs) for growing graphs. Of particular interest are growing Cayley graphs. For example, generators of free groups give rise to free independent random variables in the sense of Voiculescu, and we obtain the Wigner semicircle law; cf. [42]. Another important class of graphs to consider here is that of distanceregular graphs [5, 6, 8, 10], which generalize distance-transitive (i.e., two-point homogeneous) graphs. Among many other applications and links, these graphs have been often used as test instances for problems related to random walks on general graphs; see [10] and the references therein. Hora [16] proved CLTs for several families of distance-regular graphs, including the Hamming graphs (which are also Cayley graphs) and the Johnson graphs, and obtained various distributions, such as the Gaussian, Poisson, geometric, and the exponential distributions. The CLTs in [16] are with respect to the vacuum state, and Hora [17] then considered the Gibbs state, also known as the deformed vacuum state, and extended the CLT for the Johnson graphs and the Hamming graphs. Later it turned out that distance-regular graphs are particularly well-suited for the method of quantum decomposition of the adjacency matrix, which has been playing a key role in obtaining CLTs. This method was first introduced by Hashimoto [13] for 
certain growing Cayley graphs, and then developed and reformulated further by Hora, Obata, and others; see, e.g., [14, 15, 18, 19, 20, 29]. It not only made the whole theory transparent, but also lead to quantum central limit theorems (QCLTs), in which we take into account the three non-commuting components in the quantum decomposition.

Our goal in this paper is to establish QCLTs for those distance-regular graphs said to have classical parameters. For such a graph, the structure of the adjacency algebra is described by just three parameters denoted by $q, \alpha$, and $\beta$, together with the diameter $d$. The parameter $q$, called the base, is known to be an integer distinct from 0 and -1 , provided that $d \geqslant 3$. Having classical parameters may look like quite a strong restriction, but it is in some sense rather common among distance-regular graphs. In fact, except the cycles which we view as trivial, all the known infinite families of distance-regular graphs with unbounded diameter either have classical parameters or are closely related to those having classical parameters (by means of halving, folding, doubling, twisting, etc.); cf. [10, Section 3]. See also [33] and [32, Theorem 6.3] for geometric characterizations of this property. The classification of distance-regular graphs having classical parameters with $q=1$ is already complete, and there exist only four infinite families: the Hamming graphs, Doob graphs, halved cubes, and the Johnson graphs. These graphs were discussed by Hora [16, 17] (see also [19]) in detail, ${ }^{1}$ so we will consider the graphs with $q \neq 1$ in this paper. Our main result is Theorem 3.5 in Section 3, where we let $d \rightarrow \infty$ and determine the possible scaling limits for QCLTs in the Gibbs state, in terms of the behaviors of the classical parameters and one other parameter associated with the Gibbs state. The corresponding weak limits of the normalized spectral distributions will be described explicitly in Section 5. Currently there are fifteen known infinite families of distance-regular graphs having classical parameters with unbounded diameter, eleven of which are such that $q \neq 1$. Our results apply to these eleven families with $q \neq 1$, but we stress that they will also be equally applicable whenever we find a new such infinite family in the future. ${ }^{2}$ We may also remark that four out of the eleven families are Cayley graphs.

The contents of the other sections are as follows. In Section 2, we review basic definitions and concepts about algebraic probability spaces and distance-regular graphs, and then recall the QCLT for distance-regular graphs. Our account follows [19]. We will also sharpen the QCLT slightly (Proposition 2.6), by showing that some assumption is redundant. Section 4 is another preliminary section and is devoted to establishing formulas which are needed in Section 5. Section 6 discusses concrete examples from the eleven infinite families with $q \neq 1$ mentioned above.

\section{Algebraic probability spaces, distance-regular graphs, and the quantum central limit theorem}

An algebraic probability space is a pair $(\mathcal{A}, \varphi)$, where $\mathcal{A}$ is a $*$-algebra over $\mathbb{C}$ and $\varphi: \mathcal{A} \rightarrow \mathbb{C}$ is a state, i.e., a linear map such that $\varphi\left(1_{\mathcal{A}}\right)=1$ and that $\varphi\left(a^{*} a\right) \geqslant 0$ for every $a \in \mathcal{A}$, where $1_{\mathcal{A}}$ denotes the identity of $\mathcal{A}$; cf. [19, Section 1.1]. The elements of $\mathcal{A}$ are called (algebraic) random variables. We call $a \in \mathcal{A}$ real if $a^{*}=a$. For every real random variable $a \in \mathcal{A}$, there exists a Borel probability measure $\mu$ on $\mathbb{R}$ (cf. [7, Section 1.3]) such that

$$
\varphi\left(a^{i}\right)=\int_{-\infty}^{+\infty} \xi^{i} \mu(\mathrm{d} \xi), \quad i=0,1,2, \ldots
$$

\footnotetext{
${ }^{1}$ The Doob graphs were not mentioned in $[16,17]$, but they have the same classical parameters as certain Hamming graphs, and thus separate discussions are not necessary.

${ }^{2}$ The twisted Grassmann graphs are the last of these fifteen families and were discovered by Van Dam and Koolen [9] in 2005.
} 
We note that such a measure $\mu$ may not be unique. Several sufficient conditions are known on its uniqueness, such as Carleman's moment test; cf. [19, Theorem 1.36].

We are interested in algebraic probability spaces arising from graphs. All the graphs we consider in this paper are finite and simple. Thus, by a graph we mean a pair $\Gamma=(X, R)$ consisting of a non-empty finite set $X$ and a subset $R$ of $\left(\begin{array}{c}X \\ 2\end{array}\right)$, the set of two-element subsets of $X$. The elements of $X$ are vertices of $\Gamma$, and the elements of $R$ are edges of $\Gamma$. Two vertices $x, y \in X$ are called adjacent (and written $x \sim y$ ) if $\{x, y\} \in R$. The degree (or valency) $k(x$ ) of $x \in X$ is the number of vertices adjacent to $x$. We call $\Gamma k$-regular if $k(x)=k$ for all $x \in X$. A path of length $n$ joining $x, y \in X$ is a sequence of vertices $x=x_{0}, x_{1}, \ldots, x_{n}=y$ such that $x_{j-1} \sim x_{j}$ for $j=1,2, \ldots, n$. We will only consider connected graphs, i.e., those graphs in which any two vertices are joined by a path. The distance $\partial(x, y)$ of $x, y \in X$ is the length of a shortest path joining them. The diameter of $\Gamma$ is defined by $d=\max \{\partial(x, y): x, y \in X\}$. Let $M_{X}(\mathbb{C})$ denote the $\mathbb{C}$-algebra consisting of complex matrices with rows and columns indexed by $X$. The adjacency matrix $A$ of $\Gamma$ is the matrix in $M_{X}(\mathbb{C})$ defined by

$$
A_{x, y}=\left\{\begin{array}{ll}
1 & \text { if } x \sim y, \\
0 & \text { otherwise, }
\end{array} \quad x, y \in X .\right.
$$

By an eigenvalue of $\Gamma$, we mean an eigenvalue of $A$. Likewise, we speak of the spectrum of $\Gamma$.

As usual, we view $M_{X}(\mathbb{C})$ as a $*$-algebra by letting $*$ mean adjoint (i.e., conjugate-transpose). Associated with the graph $\Gamma$ above is the adjacency algebra $\mathcal{A}(\Gamma)$, i.e., the commutative $*$-subalgebra of $M_{X}(\mathbb{C})$ generated by $A$. Below we give three examples of states for $\mathcal{A}(\Gamma)$.

The tracial state. This is defined by

$$
\varphi_{\mathrm{tr}}(B)=\frac{1}{|X|} \operatorname{tr}(B), \quad B \in \mathcal{A}(\Gamma) .
$$

For this state, the Borel probability measure $\mu$ from (2.1) for the random variable $A$ is unique and is the spectral distribution of $\Gamma$ given by

$$
\mu\left(\theta_{i}\right)=\frac{m_{i}}{|X|}, \quad i=0,1, \ldots, e,
$$

where $\theta_{0}, \theta_{1}, \ldots, \theta_{e}$ are the distinct eigenvalues of $\Gamma$, and $m_{i}$ denotes the multiplicity of $\theta_{i}$ in the spectrum of $\Gamma$.

The vacuum state. Fix a "base vertex" $o \in X$, and let

$$
\varphi_{0}(B)=\langle\hat{o}, B \hat{o}\rangle=B_{o, o}, \quad B \in \mathcal{A}(\Gamma),
$$

where $\hat{o}$ denotes the column vector indexed by $X$ with a 1 in the $o$ coordinate and 0 in all other coordinates, and $\langle\cdot, \cdot\rangle$ denotes the standard Hermitian inner product.

The Gibbs state. This generalizes $\varphi_{0}$ above. Let $t \in \mathbb{R}$, and let

$$
\varphi_{t}(B)=\sum_{x \in X} t^{\partial(x, o)}\langle\hat{x}, B \hat{o}\rangle=\sum_{x \in X} t^{\partial(x, o)} B_{x, o}, \quad B \in \mathcal{A}(\Gamma),
$$

where $0^{0}:=1$. The Gibbs state is also called the deformed vacuum state. The scalar $-\log t$ (when $t \geqslant 0$ ) corresponds to the inverse temperature parameter in the case of the Gibbs state on a canonical ensemble. It should be remarked however that, unlike the first two examples, the Gibbs state is not always a state. ${ }^{3}$ See Lemma 2.1 below.

\footnotetext{
${ }^{3}$ For this reason, it would be more appropriate to call $\varphi_{t}$, say, the Gibbs functional.
} 
Recall that $\Gamma=(X, R)$ is assumed to be connected with diameter $d$. For every $i=0,1, \ldots, d$, let $A_{i}$ be the $i^{\text {th }}$ distance matrix of $\Gamma$, i.e.,

$$
\left(A_{i}\right)_{x, y}=\left\{\begin{array}{ll}
1 & \text { if } \partial(x, y)=i, \\
0 & \text { otherwise }
\end{array} \quad x, y \in X .\right.
$$

In particular, $A_{0}=I$ (the identity matrix) and $A_{1}=A$. We call $\Gamma$ distance-regular if there exist non-negative integers $a_{i}, b_{i}, c_{i}, i=0,1, \ldots, d$, such that $b_{d}=c_{0}=0$, and that

$$
A A_{i}=b_{i-1} A_{i-1}+a_{i} A_{i}+c_{i+1} A_{i+1}, \quad i=0,1, \ldots, d,
$$

where $b_{-1} A_{-1}=c_{d+1} A_{d+1}:=0$. We note in this case that $\Gamma$ is $k$-regular with $k=b_{0}, a_{0}=0$, $c_{1}=1$,

$$
a_{i}+b_{i}+c_{i}=k, \quad i=0,1, \ldots, d,
$$

and that $b_{i-1} c_{i} \neq 0, i=1,2, \ldots, d$. Moreover, the matrix $A_{i}$ has constant row and column sum $k_{i}$ (which is the number of vertices at distance $i$ from any given vertex), where

$$
k_{i}=\frac{b_{0} b_{1} \cdots b_{i-1}}{c_{1} c_{2} \cdots c_{i}}, \quad i=0,1, \ldots, d .
$$

Note that $k_{0}=1$ and $k_{1}=k$.

From now on, suppose that $\Gamma$ is distance-regular. It follows from (2.2) that

$$
\mathcal{A}(\Gamma)=\operatorname{span}\left\{A_{0}, A_{1}, \ldots, A_{d}\right\},
$$

from which it follows that $\operatorname{dim} \mathcal{A}(\Gamma)=d+1$, and hence that $\Gamma$ has exactly $d+1$ distinct eigenvalues $k=\theta_{0}, \theta_{1}, \ldots, \theta_{d}$. Moreover, every matrix in $\mathcal{A}(\Gamma)$ has constant diagonal entries, and hence $\varphi_{\mathrm{tr}}=\varphi_{0}$. The Gibbs state $\varphi_{t}$ is also independent of the base vertex $o \in X$, and we have

$$
\varphi_{t}(B)=\frac{1}{|X|} \operatorname{tr}\left(K_{t} B\right), \quad B \in \mathcal{A}(\Gamma)
$$

where

$$
K_{t}=\left(t^{\partial(x, y)}\right)_{x, y \in X}=A_{0}+t A_{1}+t^{2} A_{2}+\cdots+t^{d} A_{d} \in \mathcal{A}(\Gamma) .
$$

From this observation we immediately see that

Lemma 2.1. If $\Gamma$ is distance-regular, then the Gibbs state $\varphi_{t}$ is a state on $\mathcal{A}(\Gamma)$ if and only if the matrix $K_{t}$ is positive semidefinite.

It also follows from (2.2)-(2.5) that the mean and the variance of the random variable $A$ in the Gibbs state $\varphi_{t}$ are given respectively by (cf. [19, Lemma 3.25])

$$
\varphi_{t}(A)=t k, \quad \Sigma_{t}^{2}(A)=\varphi_{t}\left((A-t k I)^{2}\right)=k(1-t)\left(1+t+t a_{1}\right) .
$$

In view of Lemma 2.1, we consider the following subset of $\mathbb{R}$ :

$$
\pi(\Gamma)=\left\{t \in \mathbb{R}: K_{t} \text { is positive semidefinite }\right\} .
$$

We always have $0,1 \in \pi(\Gamma)$, so that $\pi(\Gamma) \neq \varnothing$. Moreover, by looking at the $2 \times 2$ principal submatrices of $K_{t}$, it follows that

$$
\pi(\Gamma) \subset[-1,1] .
$$


With respect to the base vertex $o \in X$, we have the following quantum decomposition of $A$ :

$$
A=A^{+}+A^{-}+A^{\circ}
$$

where

$$
\left(A^{\epsilon}\right)_{x, y}=\left\{\begin{array}{ll}
1 & \text { if } x \sim y, \partial(x, o)=\partial(y, o)+i_{\epsilon}, \\
0 & \text { otherwise }
\end{array} \quad x, y \in X\right.
$$

for $\epsilon \in\{+,-, \circ\}$, with $i_{+}=1, i_{-}=-1$, and $i_{\circ}=0$. The matrices $A^{+}, A^{-}$, and $A^{\circ}$ are called the quantum components of $A$ with respect to $o$. Consider the $\mathbb{C}$-vector space

$$
W(\Gamma)=\operatorname{span}\left\{\Phi_{0}, \Phi_{1}, \ldots, \Phi_{d}\right\},
$$

where the $\Phi_{i}$ are the unit column vectors given by

$$
\Phi_{i}=\frac{1}{\sqrt{k_{i}}} A_{i} \hat{o}, \quad i=0,1, \ldots, d .
$$

Note that $\Phi_{0}=\hat{o}$. With this notation, we have

$$
\varphi_{t}(B)=\sum_{i=0}^{d} t^{i} \sqrt{k_{i}}\left\langle\Phi_{i}, B \Phi_{0}\right\rangle, \quad B \in \mathcal{A}(\Gamma) .
$$

It follows from (2.2) and (2.4) that

$$
A^{+} \Phi_{i}=\sqrt{c_{i+1} b_{i}} \Phi_{i+1}, \quad A^{-} \Phi_{i}=\sqrt{c_{i} b_{i-1}} \Phi_{i-1}, \quad A^{\circ} \Phi_{i}=a_{i} \Phi_{i}
$$

for $i=0,1, \ldots, d$, where $\sqrt{c_{d+1} b_{d}} \Phi_{d+1}=\sqrt{c_{0} b_{-1}} \Phi_{-1}:=0$. In particular, we observe that the actions of these quantum components on $W(\Gamma)$ are independent of the base vertex $o \in X$.

Remark 2.2. The subalgebra $\tilde{\mathcal{A}}(\Gamma)$ of $M_{X}(\mathbb{C})$ generated by the quantum components $A^{+}, A^{-}$, and $A^{\circ}$ of $A$ is non-commutative unless $|X|=1$, and is contained in the Terwilliger algebra of $\Gamma$ with respect to $o$; cf. [34, 35, 36]. See [41] for discussions on when the two algebras are equal. The space $W(\Gamma)$ is an irreducible module of the Terwilliger algebra, called the primary module.

We now recall the QCLT for a growing distance-regular graph in the Gibbs state $\varphi_{t}$ established in [19, Section 3.4]. For the rest of this paper, let $\Lambda$ be an infinite directed set, and let $\left(\Gamma_{\lambda}\right)_{\lambda \in \Lambda}$ be a net of distance-regular graphs; see, e.g., [23, Chapter 2]. To simplify the notation, we will mostly omit the subscript " $\lambda$ ". We will view $X, d, k, a_{i}, b_{i}, c_{i}$, etc., as functions of $\Gamma$. The scalar $t \in \pi(\Gamma)$ is chosen and fixed for each of the $\Gamma$ so that the variance $\Sigma_{t}^{2}(A)>0$ (cf. (2.6)), and we will also think of $t$ as a function of $\Gamma$. In this paper we are mainly interested in limit distributions with infinite supports, and hence we will assume that

$$
d \rightarrow \infty
$$

(That is, $\lim _{\lambda \in \Lambda} d\left(\Gamma_{\lambda}\right)=\infty$.)

In view of $(2.6)$, we work with the following normalization when taking the limit:

$$
\frac{A-t k I}{\Sigma_{t}(A)}=\tilde{A}^{+}+\tilde{A}^{-}+\tilde{A}^{\circ}
$$

where

$$
\tilde{A}^{ \pm}=\frac{A^{ \pm}}{\Sigma_{t}(A)}, \quad \tilde{A}^{\circ}=\frac{A^{\circ}-t k I}{\Sigma_{t}(A)} .
$$


From (2.10) it follows that

$$
\tilde{A}^{+} \Phi_{i}=\sqrt{\bar{\omega}_{i+1}} \Phi_{i+1}, \quad \tilde{A}^{-} \Phi_{i}=\sqrt{\bar{\omega}_{i}} \Phi_{i-1}, \quad \tilde{A}^{\circ} \Phi_{i}=\bar{\alpha}_{i+1} \Phi_{i}
$$

for $i=0,1, \ldots, d$, where $\sqrt{\bar{\omega}_{d+1}} \Phi_{d+1}=\sqrt{\bar{\omega}_{0}} \Phi_{-1}:=0$, and

$$
\bar{\omega}_{i}=\frac{c_{i} b_{i-1}}{\Sigma_{t}^{2}(A)}, \quad i=1,2, \ldots, d, \quad \bar{\alpha}_{i}=\frac{a_{i-1}-t k}{\Sigma_{t}(A)}, \quad i=1,2, \ldots, d+1 .
$$

We also define the scalar $\bar{\gamma}_{i}$ by (cf. (2.9))

$$
\bar{\gamma}_{i}=t^{i} \sqrt{k_{i}}, \quad i=0,1, \ldots, d .
$$

Consider the following limits:

$$
\bar{\omega}_{i} \rightarrow \omega_{i}, \quad \bar{\alpha}_{i} \rightarrow \alpha_{i}, \quad i=1,2, \ldots, \quad \bar{\gamma}_{i} \rightarrow \gamma_{i}, \quad i=0,1, \ldots
$$

These limits do not necessarily exist in general, and we impose the following:

Assumption 2.3. With the above situation, we assume that the limits $\omega_{i}, \alpha_{i}$, and $\gamma_{i}$ exist and that $\omega_{i}>0$ for all $i$. We note that $\gamma_{0}=1$.

With reference to Assumption 2.3, let $\mathcal{W}$ be an infinite-dimensional $\mathbb{C}$-vector space with a fixed basis $\left\{\Psi_{i}: i=0,1, \ldots\right\}$, where we equip $\mathcal{W}$ with the Hermitian inner product $\langle\cdot, \cdot\rangle$ for which the $\Psi_{i}$ are orthonormal. We define the linear operators $B^{+}, B^{-}$, and $B^{\circ}$ on $\mathcal{W}$ by

$$
B^{+} \Psi_{i}=\sqrt{\omega_{i+1}} \Psi_{i+1}, \quad B^{-} \Psi_{i}=\sqrt{\omega_{i}} \Psi_{i-1}, \quad B^{\circ} \Psi_{i}=\alpha_{i+1} \Psi_{i}
$$

for $i=0,1, \ldots$, where $\sqrt{\omega_{-1}} \Psi_{-1}:=0$. Note that $B^{+}$and $B^{-}$are adjoints of each other. The quadruple $\left(\mathcal{W},\left\{\Psi_{i}\right\}, B^{+}, B^{-}\right)$is called the interacting Fock space (of one mode) associated with Jacobi sequence $\left\{\omega_{i}\right\}$.

Recall the non-commutative algebra $\tilde{\mathcal{A}}(\Gamma)$ from Remark 2.2 , and observe that $\tilde{A}^{+}, \tilde{A}^{-}$, and $\tilde{A}^{\circ}$ generate $\tilde{\mathcal{A}}(\Gamma)$. We now extend the domain of the Gibbs state $\varphi_{t}$ to $\tilde{\mathcal{A}}(\Gamma)$; cf. $(2.9)$. This extension is again independent of the base vertex $o \in X$, but it should be remarked that it may fail to be a state on $\tilde{\mathcal{A}}(\Gamma)$ (though it is indeed a state on $\mathcal{A}(\Gamma)$ by Lemma 2.1). The QCLT in the Gibbs state is stated as follows:

Theorem 2.4 ([19, Theorem 3.29]). With reference to Assumption 2.3, we have

$$
\varphi_{t}\left(\tilde{A}^{\epsilon_{m}} \cdots \tilde{A}^{\epsilon_{1}}\right) \rightarrow \sum_{i=0}^{\infty} \gamma_{i}\left\langle\Psi_{i}, B^{\epsilon_{m}} \cdots B^{\epsilon_{1}} \Psi_{0}\right\rangle
$$

for any $\epsilon_{1}, \ldots, \epsilon_{m} \in\{+,-, \circ\}$ and $m=1,2, \ldots$

Remark 2.5. There exists a Borel probability measure $\mu_{\infty}$ on $\mathbb{R}$ such that (cf. (2.1))

$$
\sum_{i=0}^{\infty} \gamma_{i}\left\langle\Psi_{i},\left(B^{+}+B^{-}+B^{\circ}\right)^{m} \Psi_{0}\right\rangle=\int_{-\infty}^{+\infty} \xi^{m} \mu_{\infty}(\mathrm{d} \xi), \quad m=1,2, \ldots
$$

This $\mu_{\infty}$ is called the asymptotic normalized spectral distribution of $A$ in the Gibbs state, and we are interested in finding and describing it. See Section 5.

We end this section with some comments. In [19, Section 3.4], Hora and Obata also considered the case when $\omega_{m}=0$ for some $m$, so that the probability measure $\mu_{\infty}$ above has finite support. However, if we stick to the case when $\omega_{i}>0$ for all $i$ as in Assumption 2.3, then assuming the existence of the $\gamma_{i}$ turns out to be redundant. To be more precise, we show the following: 
Proposition 2.6. Suppose that the $\omega_{i}$ and the $\alpha_{i}$ exist and that $\omega_{i}>0$ for all $i$. Then the $\gamma_{i}$ exist as well. In particular, Theorem 2.4 holds true under this weaker assumption.

Proposition 2.6 is a consequence of Claims 2.8-2.11 below. For the rest of this section, we assume the existence of the $\omega_{i}>0$ and that of the $\alpha_{i}$.

First, observe that

$$
(1-t)\left(1+t+t a_{1}\right)=\frac{\Sigma_{t}^{2}(A)}{k}=\frac{1}{\bar{\omega}_{1}} \rightarrow \frac{1}{\omega_{1}}>0 .
$$

Since $a_{0}=0$, the existence of $\gamma_{1}$ follows from that of $\alpha_{1}$ and $(2.13): \gamma_{1}=-\alpha_{1} / \sqrt{\omega_{1}}$. Note that if $k=2$ then $\Gamma$ is either the $2 d$-cycle or the $(2 d+1)$-cycle. Bang, Dubickas, Koolen, and Moulton [4] proved the Bannai-Ito conjecture:

Theorem 2.7 ([4]). There exist only finitely many distance-regular graphs for each fixed degree $k \geqslant 3$.

Since we are letting $d \rightarrow \infty$ (cf. (2.11)), it follows that

Claim 2.8. If $\xi$ is an accumulation point of $1 / k \in(0,1 / 2]$, then $\xi \in\{0,1 / 2\}$.

We next handle each of the two possible accumulation points of $1 / k$.

Claim 2.9. Suppose that $1 / 2$ is an accumulation point of $1 / k$, and consider a subnet of $\left(\Gamma_{\lambda}\right)_{\lambda \in \Lambda}$ for which $k=2$ eventually. Then the $\gamma_{i}$ exist on this subnet. Moreover, we have $\omega_{i}=\omega_{1} / 2$ and $\alpha_{i}=\alpha_{1}$ for $i=2,3, \ldots$.

Proof. Recall that $\gamma_{1}$ exists. Since $k=2$ eventually, this means that $t$ is convergent on this subnet. For the cycles, we have $k_{i}=2, i=1,2, \ldots, d-1$. Since $d \rightarrow \infty$, it follows that the $\gamma_{i}$ all exist on this subnet. The last statement also follows from (2.13) and since the cycles satisfy $\left(a_{i}, b_{i}, c_{i}\right)=(0,1,1)$ for $i=1,2, \ldots, d-1$.

Claim 2.10. Suppose that 0 is an accumulation point of $1 / k$, and consider a subnet of $\left(\Gamma_{\lambda}\right)_{\lambda \in \Lambda}$ for which $k \rightarrow \infty$. Then the $\gamma_{i}$ exist on this subnet. Moreover, we have $a_{i} / \sqrt{k} \rightarrow \alpha_{i+1} / \sqrt{\omega_{1}}+\gamma_{1}$, $b_{i} / k \rightarrow 1$, and $c_{i} \rightarrow \omega_{i} / \omega_{1}$ for $i=1,2, \ldots$ on this subnet.

Proof. That $a_{i} / \sqrt{k} \rightarrow \alpha_{i+1} / \sqrt{\omega_{1}}+\gamma_{1}, i=1,2, \ldots$, is immediate from (2.13). We next show that $b_{i} / k \rightarrow 1$ and $c_{i} \rightarrow \omega_{i} / \omega_{1}$ for $i=1,2, \ldots$ on this subnet. Suppose by induction that $c_{i} \rightarrow \omega_{i} / \omega_{1}$ for some $i$. We have $a_{i}=o(k)$ and $c_{i}=o(k)$ since $k \rightarrow \infty$, and hence $b_{i} / k \rightarrow 1$ by (2.3). Then it follows in turn that $c_{i+1} \sim \overline{\omega_{i+1}} / \overline{\omega_{1}} \rightarrow \omega_{i+1} / \omega_{1}$. The existence of the $\gamma_{i}$ on this subnet now follows from these comments, (2.4), and the existence of $\gamma_{1}$.

Finally, we show that the above two cases do not coexist.

Claim 2.11. There exists exactly one accumulation point of $1 / k \in(0,1 / 2]$. More precisely, we have either $k=2$ eventually, or $k \rightarrow \infty$.

Proof. In view of Claim 2.8, suppose on the contrary that both 0 and $1 / 2$ are accumulation points of $1 / k$. On the one hand, we have $\omega_{i}=\omega_{1} / 2, i=2,3, \ldots$, by Claim 2.9. On the other hand, we have $\omega_{i} \geqslant \omega_{1}, i=2,3, \ldots$, by Claim 2.10 and since $c_{i} \geqslant 1$. This is a contradiction, and the result follows.

Proof of Proposition 2.6. Immediate from Claims 2.8-2.11.

The following is another important consequence of the above discussions: 
Claim 2.12. Each of the $c_{i}$ is eventually constant.

Proof. Follows from Claims 2.8-2.11 and since the $c_{i}$ are integers.

Remark 2.13. In [19, Chapter 7], Hora and Obata extended the method of the quantum decomposition and the QCLT to more general growing regular graphs, and obtained some sufficient conditions for the theorem to hold. See also [20]. In particular, these conditions can be applied to Cayley graphs on Coxeter groups, such as the symmetric groups. For distance-regular graphs, these conditions turn out to be reduced to the following (besides that $\Sigma_{t}^{2}(A)>0$ ): (i) $k \rightarrow \infty$; (ii) each of the $c_{i}$ is eventually constant; (iii) the $a_{i} / \sqrt{k}$ are convergent; (iv) $\gamma_{1}$ exists. See [19, Theorem 7.14 and Proposition 7.17]. Therefore, if we focus only on distance-regular graphs with $k \geqslant 3$, then it follows from Claims 2.8-2.12 that these sufficient conditions are in fact equivalent to Assumption 2.3 (or the existence of the $\omega_{i}>0$ and that of the $\alpha_{i}$, by virtue of Proposition 2.6).

\section{Distance-regular graphs with classical parameters}

A distance-regular graph $\Gamma$ with diameter $d$ is said to have classical parameters $(d, q, \alpha, \beta)$ (cf. [8, Section 6.1]) whenever the $b_{i}$ and the $c_{i}$ are expressed as

$$
b_{i}=\left(\left[\begin{array}{l}
d \\
1
\end{array}\right]-\left[\begin{array}{l}
i \\
1
\end{array}\right]\right)\left(\beta-\alpha\left[\begin{array}{l}
i \\
1
\end{array}\right]\right), \quad c_{i}=\left[\begin{array}{l}
i \\
1
\end{array}\right]\left(1+\alpha\left[\begin{array}{c}
i-1 \\
1
\end{array}\right]\right)
$$

for $i=0,1, \ldots, d$, where

$$
\left[\begin{array}{l}
i \\
1
\end{array}\right]=1+q+q^{2}+\cdots+q^{i-1}
$$

is a Gaussian binomial coefficient. We call $q$ the base. In particular,

$$
k=b_{0}=\left[\begin{array}{l}
d \\
1
\end{array}\right] \beta,
$$

and by (2.3) we have

$$
a_{i}=\left[\begin{array}{l}
i \\
1
\end{array}\right]\left(\beta-1+\alpha\left(\left[\begin{array}{l}
d \\
1
\end{array}\right]-\left[\begin{array}{l}
i \\
1
\end{array}\right]-\left[\begin{array}{c}
i-1 \\
1
\end{array}\right]\right)\right), \quad i=0,1, \ldots, d .
$$

It is known (see [8, Proposition 6.2.1]) that

$$
q \in \mathbb{Z} \backslash\{0,-1\} \quad \text { if } \quad d \geqslant 3 .
$$

As mentioned in Section 1, all the graphs with $q=1$ are known, and the QCLTs for them have been obtained, so our aim in this paper is to discuss the case where $q \in\{ \pm 2, \pm 3, \ldots\}$.

Suppose that $\Gamma$ has classical parameters $(d, q, \alpha, \beta)$. By [8, Corollary 8.4.2], the $d+1$ distinct eigenvalues of $\Gamma$ are given by

$$
\theta_{i}=\frac{b_{i}}{q^{i}}-\left[\begin{array}{l}
i \\
1
\end{array}\right]=\left[\begin{array}{c}
d-i \\
1
\end{array}\right]\left(\beta-\alpha\left[\begin{array}{l}
i \\
1
\end{array}\right]\right)-\left[\begin{array}{l}
i \\
1
\end{array}\right], \quad i=0,1, \ldots, d .
$$

For $i=0,1, \ldots, d$, let $E_{i}$ denote the orthogonal projection onto the eigenspace of $A$ for $\theta_{i}$. The $E_{i}$ are polynomials in $A$, and we have

$$
\mathcal{A}(\Gamma)=\operatorname{span}\left\{E_{0}, E_{1}, \ldots, E_{d}\right\} .
$$


Note by (3.2) that $\theta_{0}=k$. Since $\Gamma$ is regular and connected, it follows that (cf. [8, p. 45])

$$
E_{0}=\frac{1}{|X|} J
$$

where $J$ denotes the all-ones matrix.

Recall the set $\pi(\Gamma)$ from (2.7). It seems to be a difficult problem to determine $\pi(\Gamma)$ in general. For the Hamming graphs and the Johnson graphs, which have classical parameters with $q=1$, it is shown (see [19, Propositions 5.16 and 6.27]) that this set contains the interval [0,1], as consequences of Bożejko's quadratic embedding test; cf. [19, Proposition 2.14]. For the case $q \neq 1$, the following result again finds infinitely many elements of $\pi(\Gamma)$ :

Proposition 3.1. Suppose that $\Gamma$ has classical parameters $(d, q, \alpha, \beta)$ with $d \geqslant 3$ and $q \in$ $\{ \pm 2, \pm 3, \ldots\}$. Then $q^{-i} \in \pi(\Gamma)$ for $i=0,1,2, \ldots$.

Proof. We already mentioned that $q^{0}=1 \in \pi(\Gamma)$. By [8, Corollary 8.4.2], the first projection matrix $E_{1}$ is of the form

$$
E_{1}=\frac{1}{|X|} \sum_{i=0}^{d}\left(\zeta+\eta q^{-i}\right) A_{i}=\zeta E_{0}+\frac{\eta}{|X|} K_{q^{-1}}
$$

for some $\zeta, \eta \in \mathbb{R}$, where we have used (3.6). It is customary to write $\theta_{i}^{*}=\zeta+\eta q^{-i}, i=$ $0,1, \ldots, d$. Note that $\theta_{0}^{*}=\operatorname{tr}\left(E_{1}\right)=m_{1}$, the multiplicity of $\theta_{1}$ in the spectrum of $\Gamma$. It is known (see [8, Lemma 2.2.1]) that $\left|\theta_{i}^{*}\right| \leqslant m_{1}$ for $i=0,1, \ldots, d$. We have $\eta \neq 0$, for otherwise $E_{1}$ would be a scalar multiple of $E_{0}$, a contradiction. If $\eta<0$ then $\eta q^{-1}>\eta$, so that $\theta_{1}^{*}>m_{1}$, again a contradiction. Hence $\eta>0$.

We next show that $\zeta \leqslant 0$. If $\zeta>0$ and $q \geqslant 2$ then $E_{1}$ would be a non-zero non-negative matrix and thus $\operatorname{tr}\left(E_{0} E_{1}\right)>0$ by (3.6), which is absurd. Hence suppose that $q \leqslant-2$. We observe that $\zeta \leqslant 0$ if and only if $\theta_{1}^{*} \leqslant m_{1} / q$. By [8, Lemma 2.2.1], we have $\theta_{1} / k=\theta_{1}^{*} / m_{1}$. Using this, (3.2), (3.3), and (3.4), we easily verify that $\zeta \leqslant 0$ if and only if $\theta_{1} \leqslant k / q$ if and only if $a_{1}+q+1 \leqslant 0$. A kite of length two in $\Gamma$ is a quadruple $(x, y, z, w)$ of vertices such that $x \sim y \sim w, x \sim z \sim w$, $y \sim z$, and $x \nsim w:$

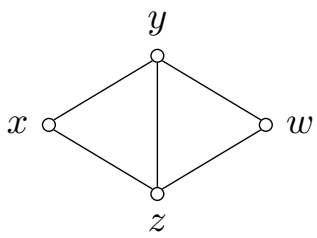

A kite of length two is also called a parallelogram of length two. By [37, Theorem 2.12], $\Gamma$ has no kite of length two. By [43, Lemma 3.6], we then have $a_{1}+q+1 \leqslant 0$. It follows that $\zeta \leqslant 0$.

Since

$$
K_{q^{-1}}=\frac{|X|}{\eta}\left(E_{1}-\zeta E_{0}\right)
$$

it follows that $K_{q^{-1}}$ is positive semidefinite, i.e., $q^{-1} \in \pi(\Gamma)$. For $i=2,3, \ldots$, we observe that the matrix $K_{q^{-i}}$ is a principal submatrix of $\left(K_{q^{-1}}\right)^{\otimes i}$ since $\left(K_{q^{-i}}\right)_{x, y}=\left(\left(K_{q^{-1}}\right)_{x, y}\right)^{i}$ for all $x, y \in X$, and it is therefore positive semidefinite as well. This completes the proof.

\footnotetext{
${ }^{4}$ The *-notation here is used to mean "dual" objects, and is standard in the theory of distance-regular graphs. The $\theta_{i}^{*}$ are referred to as the dual eigenvalues of $\Gamma$.
} 
We comment on the uniqueness of the classical parameters for $\Gamma$. By [8, Corollary 6.2.2], the classical parameters $(d, q, \alpha, \beta)$ for $\Gamma$ are uniquely determined provided that $d \geqslant 3$, with the exception of the pairs

$$
\left(d, \ell^{2}, 0, \ell\right), \quad\left(d,-\ell, \frac{\ell(\ell+1)}{1-\ell}, \frac{\ell\left(1+(-\ell)^{d}\right)}{1-\ell}\right)
$$

where $\ell \geqslant 2$. Ivanov and Shpectorov [22] showed that if $\Gamma$ has the above classical parameters then $\ell$ is a prime power and $\Gamma$ is the Hermitian dual polar graph ${ }^{2} A_{2 d-1}(\ell)$ (cf. [8, Section 9.4]).

Assumption 3.2. Recall Assumption 2.3. We moreover assume that the graph $\Gamma=\Gamma_{\lambda}$ has classical parameters $(d, q, \alpha, \beta)$ with $d \geqslant 3$ and $q \in\{ \pm 2, \pm 3, \ldots\}$. We will view $q, \alpha$, and $\beta$ as functions of $\Gamma$. For the classical parameters in (3.7), we understand that we may choose either set of them.

Recall that we are assuming that $d \rightarrow \infty$; cf. (2.11). Our goal is to describe the limit behaviors of the other parameters $q, \alpha$, and $\beta$. In the following discussions, we will freely use the expressions (3.1), (3.2), and (3.3). In particular, we note that

$$
\alpha=\frac{c_{2}}{q+1}-1 \in \frac{1}{q+1} \mathbb{Z} .
$$

The cycles $(k=2)$ with $d \geqslant 3$ do not have classical parameters, so that it follows from Claim 2.11 that

$$
k \rightarrow \infty \text {. }
$$

Claim 3.3. With reference to Assumption 3.2, limsup $|q|<\infty$. In particular, q eventually takes only finitely many values.

Proof. Suppose that $\lim \sup |q|=\infty$, or equivalently, 0 is an accumulation point of $1 / q$. Then there exists a subnet of $\left(\Gamma_{\lambda}\right)_{\lambda \in \Lambda}$ for which $|q| \rightarrow \infty$. By Claim 2.12, $c_{2}$ is eventually constant, so that it follows from (3.8) that $\alpha \rightarrow-1$ on this subnet. This in turn implies that $c_{3} \rightarrow \infty$ on this subnet, but this is impossible since $c_{3}$ is also eventually constant by Claim 2.12. It follows that $\lim \sup |q|<\infty$.

Claim 3.4. With reference to Assumption 3.2, suppose that $q$ is not convergent. Then the set of accumulation points of $q$ is of the form $\left\{\ell, \ell^{2}\right\}$ or $\left\{\ell,-\ell, \ell^{2}\right\}$ for some $\ell \in\{ \pm 2, \pm 3, \ldots\}$, where $\alpha \neq 0$ when $q= \pm \ell$, and $\alpha=0$ when $q=\ell^{2}$. Moreover, we have $a_{i} / \sqrt{k} \rightarrow 0$ for every $i=1,2, \ldots$.

Proof. Recall Claim 3.3. Since $c_{2}$ is eventually constant by Claim 2.12, it follows from (3.8) that $\alpha$ is eventually determined by $q$. We have

$$
\frac{c_{i+1}}{c_{i}}=\frac{q^{i+1}-1}{q^{i}-1} \cdot \frac{q-1+\alpha\left(q^{i}-1\right)}{q-1+\alpha\left(q^{i-1}-1\right)}, \quad i=1,2, \ldots
$$

For sufficiently large $i$ (cf. (2.11)), the RHS can be arbitrarily close to $q^{2}$ when $\alpha \neq 0$ and $q$ when $\alpha=0$. Let $\ell$ and $\ell^{\prime}$ be two distinct accumulation points of $q$, where $\left|\ell^{\prime}\right| \geqslant|\ell|(\geqslant 2)$. Since the LHS above is eventually constant for every $i$ by Claim 2.12, it follows that $\ell^{\prime} \in\left\{-\ell, \ell^{2}\right\}$, where $\alpha \neq 0$ when $q= \pm \ell$, and $\alpha=0$ when $q=\ell^{2}$.

We next show that $a_{i} / \sqrt{k} \rightarrow 0$ for every $i$. Recall that $k \rightarrow \infty$ (cf. (3.9)). The $a_{i} / \sqrt{k}$ are convergent by Claims 2.10 and 2.11. Suppose that $a_{i} / \sqrt{k} \nrightarrow 0$ for some $i$. Then we have $a_{i} \rightarrow \infty$ for this $i$, and since $q$ and $\alpha$ are eventually bounded, it follows that $\left|\beta+\alpha\left[\begin{array}{l}d \\ 1\end{array}\right]\right|=\Theta\left(a_{i}\right) \rightarrow \infty$, and 
hence that $a_{j} \sim\left[\begin{array}{l}j \\ 1\end{array}\right]\left(\beta+\alpha\left[\begin{array}{l}d \\ 1\end{array}\right]\right) \sim\left[\begin{array}{l}j \\ 1\end{array}\right] a_{1}$ for all $j$. On the one hand, this shows that $a_{1} / \sqrt{k} \nrightarrow 0$. On the other hand, this also shows that $a_{j} / \sqrt{k}$ cannot converge whenever $j \geqslant 2$ since $\left[\begin{array}{l}j \\ 1\end{array}\right]$ takes at least two values depending on $q$. Hence we must have $a_{i} / \sqrt{k} \rightarrow 0$ for every $i$, as desired.

It remains to show that $\ell^{2}$ is an accumulation point of $q$. There is nothing to prove if $\ell^{\prime}=\ell^{2}$, so that we assume that $\ell^{\prime}=-\ell$. We have $c_{3}=\left(q^{2}+q+1\right)\left(c_{2}-q\right)$ by (3.8). By setting $q= \pm \ell$ in this expression and then equating, we find that eventually $c_{2}=\ell^{2}+1$. Choose $q \in\{\ell,-\ell\}$ with $q<0$, and recall that $\alpha \neq 0$ in this case. In particular, we have $a_{2} \neq\left[\begin{array}{l}2 \\ 1\end{array}\right] a_{1}$. Suppose that $a_{1}=0$. Then $a_{2} \neq 0$, and we have $c_{2} \leqslant 2$ by [30, Theorem 2.1], but this is impossible since $c_{2}=\ell^{2}+1 \geqslant 5$. Hence $a_{1} \neq 0$. Then it follows from [44, Main Theorem] that, provided that $d \geqslant 4$, either (i) $\Gamma$ is the dual polar graph ${ }^{2} A_{2 d-1}(-q)$ with $\alpha=q(q-1) /(q+1)$ (cf. [8, Section 9.4]), or (ii) $\Gamma$ is the Hermitian forms graph Her $\left(d, q^{2}\right)$ with $\alpha=q-1$ (cf. [8, Section 9.5]), or (iii) we have $\alpha=(q-1) / 2$ and $\beta=-\left(q^{d}+1\right) / 2$. Since $c_{2}=q^{2}+1$, it follows from (3.8) that we are in (i) above. However, the graph ${ }^{2} A_{2 d-1}(-q)$ has another set of classical parameters $\left(d, q^{2}, 0,-q\right)$ (cf. (3.7)), and therefore $\ell^{2}=q^{2}$ must also be an accumulation point.

Theorem 3.5. With reference to Assumption 3.2, q eventually takes at most three values. Suppose that $q$ is eventually constant. Then so is $\alpha$, and the following hold:

(i) If $\alpha \neq 0$, then $\beta / \sqrt{k}$ is eventually bounded, and there exist scalars $\gamma$ and $\rho$ with $\rho>0$ and $\gamma(\rho+\alpha / \rho)>-1$, such that $t \sqrt{k} \rightarrow \gamma$ and the accumulation points of $\beta / \sqrt{k}$ are in $\{\rho, \alpha / \rho\}$. Moreover, we have $\rho=\sqrt{-\alpha}$ if $q<0$.

(ii) If $\alpha=0$, then there exist scalars $\gamma$ and $\rho$ with $\rho \geqslant 0$ and $\gamma \rho>-1$, such that $t \sqrt{k} \rightarrow \gamma$ and $\beta / \sqrt{k} \rightarrow \rho$.

Suppose that $q$ is not convergent. Then there exists a subnet of $\left(\Gamma_{\lambda}\right)_{\lambda \in \Lambda}$ for which $q$ is eventually constant and (ii) holds above with $\rho=0$.

Conversely, if $\left(\Gamma_{\lambda}\right)_{\lambda \in \Lambda}$ is a net of distance-regular graphs having classical parameters with $d \geqslant 3$ and $q \in\{ \pm 2, \pm 3, \ldots\}$, where $q$ and $\alpha$ are eventually constant, such that $d \rightarrow \infty$ and $(i)$ or $($ ii $)$ holds above with respect to a suitable function $t \in \pi(\Gamma)$ with $\Sigma_{t}^{2}(A)>0$, then $\left(\Gamma_{\lambda}\right)_{\lambda \in \Lambda}$ satisfies Assumption 2.3 (and thus Assumption 3.2 as well).

Proof. The first statement follows from Claims 3.3 and 3.4. We also mentioned earlier (cf. (3.9)) that $k \rightarrow \infty$.

Suppose that $q$ is eventually constant. That $\alpha$ is eventually constant follows from Claim 2.12 and (3.8). Set $\gamma=\gamma_{1}$. Since it exists, we have $t \rightarrow 0$. Recall again that the $a_{i} / \sqrt{k}$ are convergent by Claims 2.10 and 2.11, and observe that this is equivalent to saying that $\left(\beta+\alpha\left[\begin{array}{l}d \\ 1\end{array}\right]\right) / \sqrt{k}$ converges, say, to $\sigma$. Assume that $\alpha \neq 0$, and let $\xi=\rho$ be a root of the equation $\xi+\alpha / \xi=\sigma$ in the variable $\xi$. Then the other root is $\xi=\alpha / \rho$. Since $\beta / \sqrt{k}$ and $\left[\begin{array}{l}d \\ 1\end{array}\right] / \sqrt{k}$ are inverses of each other, it follows that $\beta / \sqrt{k}$ is eventually bounded, and that $\rho$ and $\alpha / \rho$ are its only possible accumulation points. If $q>0$ then $\alpha>0$ and $\beta>0$, so that we must have $\rho>0$. If $q<0$ then $\alpha<0$, and since $a_{i} / \sqrt{k} \rightarrow\left[\begin{array}{l}i \\ 1\end{array}\right] \sigma$ for every $i$ and the $\left[\begin{array}{l}i \\ 1\end{array}\right]$ alternate in sign, it follows that $\sigma=0$, so that we may take $\rho=\sqrt{-\alpha}>0$ (and thus $\alpha / \rho=-\sqrt{-\alpha}<0$ ). By (2.13) and since $t a_{1}=t \sqrt{k} \cdot a_{1} / \sqrt{k} \rightarrow \gamma \sigma=\gamma(\rho+\alpha / \rho)$, we have $\gamma(\rho+\alpha / \rho)>-1$. Assume next that $\alpha=0$. We have $q>0$ and $\beta>0$ in this case, and set $\rho=\sigma \geqslant 0$.

Suppose that $q$ is not convergent, and let the integer $\ell$ be as in Claim 3.4. Then $\ell^{2}$ is an accumulation point of $q$, so that there is a subnet of $\left(\Gamma_{\lambda}\right)_{\lambda \in \Lambda}$ for which eventually $q=\ell^{2}$. Recall by Claim 3.4 that eventually $\alpha=0$, and that $a_{i} / \sqrt{k} \rightarrow 0$ for every $i$. Hence we are in the second case above with $\rho=\sigma=0$.

Finally, let $\left(\Gamma_{\lambda}\right)_{\lambda \in \Lambda}$ be a net of distance-regular graphs as described in the last paragraph of the theorem. Note that $k \geqslant 3$ since the cycles with $d \geqslant 3$ do not have classical parameters. Hence it follows from Theorem 2.7 that $k \rightarrow \infty$. Since $t \sqrt{k} \rightarrow \gamma$, we then have $t \rightarrow 0$. If $\alpha \neq 0$ then 
we must also have $|\beta| \rightarrow \infty$ since $\rho$ and $\alpha / \rho$ are non-zero. Observe that $a_{i} / \sqrt{k} \rightarrow\left[{ }_{1}^{i}\right](\rho+\alpha / \rho)$ for every $i$, where we set $0 / 0:=0$ for brevity. In particular, we have $t a_{1} \rightarrow \gamma(\rho+\alpha / \rho)>-1$, from which it follows that $\omega_{1}$ exists and is positive. It is now immediate to verify that the $\omega_{i}$ all exist and are positive, and that the $\alpha_{i}$ exist. From Proposition 2.6 it also follows that the $\gamma_{i}$ exist.

Consider the case when $q$ is eventually constant in Theorem 3.5, and recall that so is $\alpha$ in this case. Recall (cf. (3.1)) also the formula for the $c_{i}$. The scalars $\omega_{i}, \alpha_{i}$, and $\gamma_{i}$ are expressed in terms of $q, \alpha$, and the two scalars $\gamma$ and $\rho$ in Theorem 3.5 (i) and $(i i)$ as

$$
\omega_{i}=\frac{c_{i}}{1+\gamma(\rho+\alpha / \rho)}, \quad \alpha_{i}=\frac{\left[\begin{array}{c}
i-1 \\
1
\end{array}\right](\rho+\alpha / \rho)-\gamma}{\sqrt{1+\gamma(\rho+\alpha / \rho)}}, \quad i=1,2, \ldots,
$$

and

$$
\gamma_{i}=\frac{\gamma^{i}}{\sqrt{c_{i} \cdot c_{1}}}, \quad i=0,1, \ldots
$$

where we set $0 / 0:=0$ and $0^{0}:=1$.

\section{More background on graphs with classical parameters}

In order to describe the asymptotic normalized spectral distributions corresponding to Theorem 3.5 $(i)$ and $(i i)$, we collect in this section necessary formulas for distance-regular graphs with classical parameters. Thus, throughout this section, we let $\Gamma=(X, R)$ denote a (fixed) distance-regular graph with classical parameters $(d, q, \alpha, \beta)$, where $d \geqslant 3$ and $q \in\{ \pm 2, \pm 3, \ldots\}$.

Recall the eigenvalues $\theta_{i}, i=0,1, \ldots, d$, of $\Gamma$ and the corresponding orthogonal projections $E_{i}$, $i=0,1, \ldots, d$; cf. (3.4) and (3.5). It is known (see [8, Corollary 8.4.2]) that the ordering $\left(E_{0}, E_{1}, \ldots, E_{d}\right)$ is $Q$-polynomial; that is to say, there exist scalars ${ }^{5} a_{i}^{*}, b_{i}^{*}, c_{i}^{*} \in \mathbb{R}, i=0,1, \ldots, d$, such that $b_{d}^{*}=c_{0}^{*}=0, b_{i-1}^{*} c_{i}^{*} \neq 0, i=1,2, \ldots, d$, and that

$$
E_{1} \circ E_{i}=\frac{1}{|X|}\left(b_{i-1}^{*} E_{i-1}+a_{i}^{*} E_{i}+c_{i+1}^{*} E_{i+1}\right), \quad i=0,1, \ldots, d,
$$

where $\circ$ denotes the entrywise (or Hadamard or Schur) product of matrices, and $b_{-1}^{*} E_{-1}=$ $c_{d+1}^{*} E_{d+1}:=0$. Note that this property is dual to (2.2). See [10, Section 5] for recent updates on the study of $Q$-polynomial distance-regular graphs.

From (2.2) it follows that there exist polynomials $v_{i}(\xi) \in \mathbb{R}[\xi], i=0,1, \ldots, d$, such that $\operatorname{deg} v_{i}(\xi)=i$ and $A_{i}=v_{i}(A)$. Set $u_{i}(\xi)=v_{i}(\xi) / k_{i}, i=0,1, \ldots, d$. In general, by Leonard's theorem (see [26], [5, Section III.5]), the polynomials $u_{i}$ associated with every $Q$-polynomial distance-regular graph are expressed in terms of the q-Racah polynomials (cf. [25, Section 3.2]) and their special/limit cases in the Askey scheme of (basic) hypergeometric orthogonal polynomials $[24,25]$. In the most general (i.e., $q$-Racah) case, the $u_{i}$ are of the form

$$
u_{i}\left(\theta_{j}\right)={ }_{4} \phi_{3}\left(\begin{array}{c}
q^{-i}, s^{*} q^{i+1}, q^{-j}, s q^{j+1} \\
r_{1} q, r_{2} q, q^{-d}
\end{array} \mid q ; q\right), \quad i, j=0,1, \ldots, d,
$$

where the parameters $r_{1}, r_{2}, s$, and $s^{*}$ satisfy $r_{1} r_{2}=s s^{*} q^{d+1} \neq 0$, and we are using the standard notation for a basic hypergeometric series ${ }_{m} \phi_{n}$ :

$$
{ }_{m} \phi_{n}\left(\begin{array}{c}
\mathfrak{a}_{1}, \ldots, \mathfrak{a}_{m} \\
\mathfrak{b}_{1}, \ldots, \mathfrak{b}_{n}
\end{array} \mid q ; \mathfrak{x}\right)=\sum_{h=0}^{\infty} \frac{\left(\mathfrak{a}_{1} ; q\right)_{h} \cdots\left(\mathfrak{a}_{m} ; q\right)_{h}}{\left(\mathfrak{b}_{1} ; q\right)_{h} \cdots\left(\mathfrak{b}_{n} ; q\right)_{h}} \frac{(-1)^{(m-n-1) h} \mathfrak{x}^{h}}{(q ; q)_{h} q^{(m-n-1)\left(\begin{array}{c}
h \\
2
\end{array}\right)}}
$$

\footnotetext{
${ }^{5}$ See footnote 4 .
} 
where $(\mathfrak{a} ; q)_{h}$ denotes the $q$-shifted factorial defined by

$$
(\mathfrak{a} ; q)_{h}=(1-\mathfrak{a})(1-\mathfrak{a} q) \cdots\left(1-\mathfrak{a} q^{h-1}\right), \quad h=0,1, \ldots
$$

To get the $u_{i}$ for our $\Gamma$, first fix $s, r_{2} \neq 0$ and let $s^{*} \rightarrow 0$ (so $r_{1} \rightarrow 0$ ), and then set

$$
s=\frac{\alpha+1-q}{(\alpha-\beta+\beta q) q^{d+1}}, \quad r_{2}=\frac{\alpha}{(\alpha-\beta+\beta q) q},
$$

or equivalently,

$$
\alpha=\frac{r_{2}(1-q)}{s q^{d}-r_{2}}, \quad \beta=\frac{r_{2} q-1}{q\left(s q^{d}-r_{2}\right)} .
$$

See [32, Proposition 6.2]. The $u_{i}$ are the dual q-Hahn polynomials (cf. [25, Section 3.7]) for $s \neq 0$ and $r_{2} \neq 0$, the affine $q$-Krawtchouk polynomials (cf. [25, Section 3.16]) for $s=0$ and $r_{2} \neq 0$, and the dual $q$-Krawtchouk polynomials (cf. [25, Section 3.17]) for $s \neq 0$ and $r_{2}=0$. See also [40, Examples 5.3-5.9]. We note that, in [32, Proposition 6.2], there is mentioned another case, called IA, which also corresponds to classical parameters with $q \neq 1$. The $u_{i}$ are then the quantum q-Krawtchouk polynomials (cf. [25, Section 3.14]). However, it is known (see [10, Proposition 5.8]) that there exists no actual $\Gamma$ in this case.

For later use, we now establish another basic hypergeometric expression for the polynomials $u_{i}$. For the moment, fix $i, j=0,1, \ldots, d$. Recall Sear's transformation formula for a terminating balanced ${ }_{4} \phi_{3}$ series (cf. [25, Section 0.6]):

$$
{ }_{4} \phi_{3}\left(\begin{array}{c}
q^{-i}, \mathfrak{x}, \mathfrak{y}, \mathfrak{z} \\
\mathfrak{l}, \mathfrak{m}, \mathfrak{n}
\end{array} \mid q ; q\right)={ }_{4} \phi_{3}\left(\begin{array}{c}
q^{-i}, \mathfrak{x}, \mathfrak{l} / \mathfrak{y}, \mathfrak{l} / \mathfrak{z} \\
\mathfrak{l}, \mathfrak{x} q^{1-i} / \mathfrak{m}, \mathfrak{x} q^{1-i} / \mathfrak{n}
\end{array} \mid q ; q\right) \frac{(\mathfrak{m} / \mathfrak{x} ; q)_{i}(\mathfrak{n} / \mathfrak{x} ; q)_{i}}{(\mathfrak{m} ; q)_{i}(\mathfrak{n} ; q)_{i}} \mathfrak{x}^{i}
$$

where $\mathfrak{x} \mathfrak{y} \mathfrak{z} q^{1-i}=\mathfrak{l m} \mathfrak{n} \neq 0$. Applying this formula twice and then simplifying a bit, we obtain

$$
{ }_{4} \phi_{3}\left(\begin{array}{c}
q^{-i}, \mathfrak{x}, \mathfrak{y}, \mathfrak{z} \\
\mathfrak{l}, \mathfrak{m}, \mathfrak{n}
\end{array} \mid q ; q\right)={ }_{4} \phi_{3}\left(\begin{array}{c}
q^{-i}, \mathfrak{x}, \mathfrak{x} \mathfrak{y} q^{1-i} / \mathfrak{l n}, \mathfrak{x} \mathfrak{z} q^{1-i} / \mathfrak{l n} \\
\mathfrak{x} q^{1-i} / \mathfrak{n}, \mathfrak{x} q^{1-i} / \mathfrak{l}, \mathfrak{m}
\end{array} \mid q ; q\right) \frac{(\mathfrak{n} / \mathfrak{x} ; q)_{i}(\mathfrak{l} / \mathfrak{x} ; q)_{i}}{(\mathfrak{n} ; q)_{i}(\mathfrak{l} ; q)_{i}} \mathfrak{x}^{i}
$$

Set

$$
\mathfrak{x}=q^{-j}, \quad \mathfrak{y}=s^{*} q^{i+1}, \quad \mathfrak{z}=s q^{j+1}, \quad \mathfrak{l}=q^{-d}, \quad \mathfrak{m}=r_{1} q, \quad \mathfrak{n}=r_{2} q
$$

in this result. Then we obtain the following expression for the $u_{i}$ for the $q$-Racah case:

$$
u_{i}\left(\theta_{j}\right)={ }_{4} \phi_{3}\left(\begin{array}{c}
q^{-i}, q^{-j}, s^{*} q^{d-j+1} / r_{2}, s q^{d-i+1} / r_{2} \\
q^{-i-j} / r_{2}, q^{d-i-j+1}, r_{1} q
\end{array} \mid q ; q\right) \frac{\left(r_{2} q^{j+1} ; q\right)_{i}\left(q^{j-d} ; q\right)_{i}}{\left(r_{2} q ; q\right)_{i}\left(q^{-d} ; q\right)_{i} q^{i j}} .
$$

By letting $s^{*} \rightarrow 0$, the RHS becomes

$$
\begin{aligned}
{ }_{3} \phi_{2}\left(\begin{array}{c}
q^{-i}, q^{-j}, s q^{d-i+1} / r_{2} \\
q^{-i-j} / r_{2}, q^{d-i-j+1}
\end{array} \mid q ; q\right) \frac{\left(r_{2} q^{j+1} ; q\right)_{i}\left(q^{j-d} ; q\right)_{i}}{\left(r_{2} q ; q\right)_{i}\left(q^{-d} ; q\right)_{i} q^{i j}} \\
\quad=\sum_{h=0}^{i} \frac{\left(q^{-i} ; q\right)_{h}\left(q^{-j} ; q\right)_{h}\left(r_{2} ; s q^{d-i+1} ; q\right)_{h} q^{h}}{\left(r_{2} ; q^{-i-j} ; q\right)_{h}\left(q^{d-i-j+1} ; q\right)_{h}(q ; q)_{h}} \frac{\left(r_{2} q^{j+1} ; q\right)_{i}\left(q^{j-d} ; q\right)_{i}}{\left(r_{2} q ; q\right)_{i}\left(q^{-d} ; q\right)_{i} q^{i j}},
\end{aligned}
$$

where we write

$$
(\mathfrak{x} ; \mathfrak{y} ; q)_{h}=(\mathfrak{x}-\mathfrak{y})(\mathfrak{x}-\mathfrak{y} q) \cdots\left(\mathfrak{x}-\mathfrak{y} q^{h-1}\right), \quad h=0,1, \ldots
$$


for convenience. We then set $s$ and $r_{2}$ as in (4.2), and obtain the $u_{i}$ for $\Gamma$ as follows:

$$
u_{i}\left(\theta_{j}\right)=\sum_{h=0}^{i} \frac{\left(q^{-i} ; q\right)_{h}\left(q^{-j} ; q\right)_{h}\left(\alpha ;(\alpha+1-q) q^{1-i} ; q\right)_{h} q^{h}}{\left(\alpha ;(\alpha-\beta+\beta q) q^{1-i-j} ; q\right)_{h}\left(q^{d-i-j+1} ; q\right)_{h}(q ; q)_{h}} \frac{\left(\alpha-\beta+\beta q ; \alpha q^{j} ; q\right)_{i}\left(q^{j-d} ; q\right)_{i}}{(\alpha-\beta+\beta q ; \alpha ; q)_{i}\left(q^{-d} ; q\right)_{i} q^{i j}} .
$$

Using (2.4) and (3.1) we have

$$
k_{i}=\frac{\left(q^{-d} ; q\right)_{i}(\alpha-\beta+\beta q ; \alpha ; q)_{i} q^{d i}}{(\alpha+1-q ; \alpha ; q)_{i}(q ; q)_{i}},
$$

from which it follows that

$$
v_{i}\left(\theta_{j}\right)=k_{i} u_{i}\left(\theta_{j}\right)=\sum_{h=0}^{i} \frac{\left(q^{-j} ; q\right)_{h}\left(q^{j-d} ; q\right)_{i-h}\left(\alpha-\beta+\beta q ; \alpha q^{j} ; q\right)_{i-h} q^{(i-h)(d-j)+j h}}{(q ; q)_{h}(\alpha+1-q ; \alpha ; q)_{i-h}(q ; q)_{i-h}} .
$$

Let $m_{i}=\operatorname{tr}\left(E_{i}\right)$, the multiplicity of $\theta_{i}$ in the spectrum of $\Gamma$. This value is computed in [8, Theorem 8.4.3]:

$$
\begin{aligned}
m_{i}= & \frac{\prod_{h=0}^{i-1}\left[\begin{array}{c}
d-h \\
1
\end{array}\right]\left(\beta-\left[\begin{array}{c}
h \\
1
\end{array}\right] \alpha\right)\left(1+\left[\begin{array}{c}
d-h \\
1
\end{array}\right] \alpha+q^{d-h} \beta\right)}{\prod_{h=1}^{i}\left[\begin{array}{c}
h \\
1
\end{array}\right]\left(\beta-\left[\begin{array}{c}
h \\
1
\end{array}\right] \alpha+q^{h}\right)\left(1+\left[\begin{array}{c}
d-h \\
1
\end{array}\right] \alpha\right)} \frac{\left(1+\left[\begin{array}{c}
d-2 i \\
1
\end{array}\right] \alpha+q^{d-2 i} \beta\right) q^{i}}{1+\left[\begin{array}{l}
d \\
1
\end{array}\right] \alpha+q^{d} \beta} \\
= & \frac{\left(q^{-d} ; q\right)_{i}(\alpha-\beta+\beta q ; \alpha ; q)_{i}\left(\alpha-\beta+\beta q ;(\alpha+1-q) q^{-d} ; q\right)_{i}}{(q ; q)_{i}(\alpha-\beta+\beta q ;(\alpha+1-q) q ; q)_{i}\left(\alpha+1-q ; \alpha q^{d-i} ; q\right)_{i}} \\
& \times \frac{\left(\alpha-\beta+\beta q-(\alpha+1-q) q^{2 i-d}\right) q^{2 d i-i^{2}}}{\alpha-\beta+\beta q-(\alpha+1-q) q^{-d}} .
\end{aligned}
$$

Finally, we obtain a closed formula for $|X|$, the number of vertices of $\Gamma$. Recall the $\mathbb{C}$-vector space $W(\Gamma)$ from $(2.8)$. In view of $(3.5), W(\Gamma)$ has another orthonormal basis $\Psi_{0}, \Psi_{1}, \ldots, \Psi_{d}$ defined by

$$
\Psi_{i}=\sqrt{\frac{|X|}{m_{i}}} E_{i} \hat{o}, \quad i=0,1, \ldots, d .
$$

As in the proof of Proposition 3.1, write

$$
E_{1}=\frac{1}{|X|} \sum_{i=0}^{d} \theta_{i}^{*} A_{i}
$$

Now, let

$$
D=|X| \operatorname{diag} E_{1} \hat{o} .
$$

Then we have

$$
A \Psi_{i}=\theta_{i} \Psi_{i}, \quad D \Phi_{i}=\theta_{i}^{*} \Phi_{i}, \quad i=0,1, \ldots, d .
$$

Moreover, it follows from (2.2) and (4.1) that the matrix representing the action of $A$ (resp. $D$ ) on $W(\Gamma)$ with respect to the $\Phi_{i}$ (resp. the $\Psi_{i}$ ) is tridiagonal with non-zero superdiagonal and subdiagonal entries. This means that $A$ and $D$ act on $W(\Gamma)$ as a Leonard pair in the sense of [38, Definition 1.1]. In the theory of Leonard pairs, there is a scalar denoted by $\nu$ [39, Definition 9.3], and it is easy to see that $\nu=\left\langle\Phi_{0}, \Psi_{0}\right\rangle^{-2}=|X|$ for the above Leonard pair on $W(\Gamma)$. For the $q$-Racah case, the scalar $\nu$ is given in $[39$, p. 273] as follows:

$$
\nu=\frac{\left(s q^{2} ; q\right)_{d}\left(s^{*} q^{2} ; q\right)_{d}}{r_{1}^{d} q^{d}\left(s q / r_{1} ; q\right)_{d}\left(s^{*} q / r_{1} ; q\right)_{d}} .
$$

Again by letting $s^{*} \rightarrow 0$ and then setting $s$ and $r_{2}$ as in (4.2), it follows that

$$
|X|=\frac{(-1)^{d}\left(\alpha-\beta+\beta q ;(\alpha+1-q) q^{1-d} ; q\right)_{d} q^{\left(\begin{array}{l}
d \\
2
\end{array}\right)}}{(\alpha+1-q ; \alpha ; q)_{d}} .
$$




\section{Description of asymptotic normalized spectral distributions}

In this section, we describe the asymptotic normalized spectral distributions (cf. Remark 2.5) corresponding to Theorem $3.5(i)$ and $(i i)$, following [17].

We retain the notation of the previous section. The Borel probability measure $\mu$ on $\mathbb{R}$ from (2.1) associated with the normalized adjacency matrix (2.12) is given by

$$
\mu\left(\frac{\theta_{j}-t k}{\Sigma_{t}(A)}\right)=\sum_{i=0}^{d} t^{i} v_{i}\left(\theta_{j}\right) \frac{m_{j}}{|X|}, \quad j=0,1, \ldots, d
$$

which follows from (2.5) and since

$$
\left(\frac{A-t k I}{\Sigma_{t}(A)}\right)^{\ell} E_{j}=\left(\frac{\theta_{j}-t k}{\Sigma_{t}(A)}\right)^{\ell} E_{j}, \quad A_{i} E_{j}=v_{i}\left(\theta_{j}\right) E_{j}
$$

for $\ell=0,1, \ldots$ and $i, j=0,1, \ldots, d$. From (4.4) and (4.5) it follows that

$$
\begin{aligned}
\frac{m_{j}}{|X|}= & \frac{\left(q^{-d} ; q\right)_{j}(\alpha-\beta+\beta q ; \alpha ; q)_{j}(\alpha+1-q ; \alpha ; q)_{d-j}}{(q ; q)_{j}\left(\alpha-\beta+\beta q ;(\alpha+1-q) q^{j-d} ; q\right)_{d+1}} \\
& \times(-1)^{d}\left(\alpha-\beta+\beta q-(\alpha+1-q) q^{2 j-d}\right) q^{2 d j-j^{2}-\left(\begin{array}{c}
d \\
2
\end{array}\right)}
\end{aligned}
$$

for $j=0,1, \ldots, d$. From (4.3) it follows that

$$
\begin{aligned}
\sum_{i=0}^{d} t^{i} v_{i}\left(\theta_{j}\right) & =\sum_{h=0}^{d} \frac{\left(q^{-j} ; q\right)_{h} q^{j h} t^{h}}{(q ; q)_{h}} \sum_{i=h}^{d} \frac{\left(q^{j-d} ; q\right)_{i-h}\left(\alpha-\beta+\beta q ; \alpha q^{j} ; q\right)_{i-h} q^{(i-h)(d-j)} t^{i-h}}{(\alpha+1-q ; \alpha ; q)_{i-h}(q ; q)_{i-h}} \\
& ={ }_{1} \phi_{0}\left(\begin{array}{c}
q^{-j} \\
-
\end{array} \mid q ; q^{j} t\right) \sum_{\ell=0}^{d-j} \frac{\left(q^{j-d} ; q\right)_{\ell}\left(\alpha-\beta+\beta q ; \alpha q^{j} ; q\right)_{\ell} q^{\ell(d-j)} t^{\ell}}{(\alpha+1-q ; \alpha ; q)_{\ell}(q ; q)_{\ell}} \\
& =(t ; q)_{j} \sum_{\ell=0}^{d-j} \frac{\left(q^{j-d} ; q\right)_{\ell}\left(\alpha-\beta+\beta q ; \alpha q^{j} ; q\right)_{\ell} q^{\ell(d-j)} t^{\ell}}{(\alpha+1-q ; \alpha ; q)_{\ell}(q ; q)_{\ell}}
\end{aligned}
$$

for $j=0,1, \ldots, d$, where we have used the $q$-binomial theorem (cf. [25, Section 0.5])

$$
{ }_{1} \phi_{0}\left(\begin{array}{c}
q^{-n} \\
-
\end{array} \mid q ; \mathfrak{x}\right)=\left(\mathfrak{x} q^{-n} ; q\right)_{n}, \quad n=0,1,2, \ldots
$$

Note that the last sum in (5.3) is a $\phi_{1}$ in general.

\section{$5.1 \quad$ Case $\beta / \sqrt{k} \rightarrow \rho>0$}

With reference to Assumption 3.2, suppose that we are in Theorem 3.5 $(i)$, or $(i i)$ with $\rho>0$. For $(i)$, we moreover assume that $\rho$ is indeed an accumulation point of $\beta / \sqrt{k}$, and will consider a subnet of $\left(\Gamma_{\lambda}\right)_{\lambda \in \Lambda}$ for which $\beta / \sqrt{k} \rightarrow \rho$ if necessary (i.e., if $\alpha / \rho$ is also an accumulation point). We note that $k \rightarrow \infty$ (cf. (3.9)), $|\beta| \rightarrow \infty, t \rightarrow 0$, and that

$$
\frac{\beta(q-1)}{q^{d}} \sim \frac{\beta^{2}}{k} \rightarrow \rho^{2}, \quad t \beta=t \sqrt{k} \frac{\beta}{\sqrt{k}} \rightarrow \gamma \rho, \quad t q^{d} \rightarrow \frac{\gamma(q-1)}{\rho} .
$$


Using this, (3.4), (5.2), and (5.3), we routinely compute

$$
\begin{aligned}
& \frac{\theta_{d-j}-t k}{\Sigma_{t}(A)} \rightarrow \frac{\left[\begin{array}{l}
j \\
1
\end{array}\right]\left(\rho-\alpha / \rho q^{j}\right)-1 / \rho q^{j}-\gamma}{\sqrt{1+\gamma(\rho+\alpha / \rho)}}, \\
& \frac{m_{d-j}}{|X|} \rightarrow \frac{\left(\alpha / \rho^{2} q^{j+1} ; q^{-1}\right)_{\infty}(\alpha+1-q ; \alpha ; q)_{j}\left(1-(\alpha+1-q) / \rho^{2} q^{2 j}\right)}{\left((\alpha+1-q) / \rho^{2} q^{j} ; q^{-1}\right)_{\infty}(q ; q)_{j} \rho^{2 j} q^{j^{2}-j}}, \\
& \sum_{i=0}^{d} t^{i} v_{i}\left(\theta_{d-j}\right) \rightarrow\left(\gamma(q-1) / \rho q^{j+1} ; q^{-1}\right)_{\infty} \sum_{\ell=0}^{j} \frac{\left(q^{-j} ; q\right)_{\ell}\left(\alpha / \rho^{2} q^{j} ; q\right)_{\ell} \gamma^{\ell} \rho^{\ell} q^{j \ell}(q-1)^{\ell}}{(\alpha+1-q ; \alpha ; q)_{\ell}(q ; q)_{\ell}}
\end{aligned}
$$

for $j=0,1,2, \ldots$ The measure (5.1) converges weakly to the discrete measure $\mu_{\infty}$ on $\mathbb{R}$ defined on the limit points in (5.5), where the masses are given by the products of the limits in (5.6) and $(5.7){ }^{6}$

\section{$5.2 \quad$ Case $\alpha \neq 0, \beta / \sqrt{k} \rightarrow \alpha / \rho$}

With reference to Assumption 3.2, suppose that we are in Theorem 3.5 $(i)$, and that $\alpha / \rho$ is an accumulation point of $\beta / \sqrt{k}$. We will consider a subnet of $\left(\Gamma_{\lambda}\right)_{\lambda \in \Lambda}$ for which $\beta / \sqrt{k} \rightarrow \alpha / \rho$ if necessary. The formulas for the limit distribution are simply obtained by replacing $\rho$ by $\alpha / \rho$ in those of the previous case:

$$
\begin{aligned}
& \frac{\theta_{d-j}-t k}{\Sigma_{t}(A)} \rightarrow \frac{\left[\begin{array}{l}
j \\
1
\end{array}\right]\left(\alpha / \rho-\rho / q^{j}\right)-\rho / \alpha q^{j}-\gamma}{\sqrt{1+\gamma(\rho+\alpha / \rho)}}, \\
& \frac{m_{d-j}}{|X|} \rightarrow \frac{\left(\rho^{2} / \alpha q^{j+1} ; q^{-1}\right)_{\infty}(\alpha+1-q ; \alpha ; q)_{j}\left(1-(\alpha+1-q) \rho^{2} / \alpha^{2} q^{2 j}\right) \rho^{2 j}}{\left((\alpha+1-q) \rho^{2} / \alpha^{2} q^{j} ; q^{-1}\right)_{\infty}(q ; q)_{j} \alpha^{2 j} q^{j^{2}-j}}, \\
& \sum_{i=0}^{d} t^{i} v_{i}\left(\theta_{d-j}\right) \rightarrow\left(\gamma \rho(q-1) / \alpha q^{j+1} ; q^{-1}\right)_{\infty} \sum_{\ell=0}^{j} \frac{\left(q^{-j} ; q\right)_{\ell}\left(\rho^{2} / \alpha q^{j} ; q\right)_{\ell} \alpha^{\ell} \gamma^{\ell} q^{j \ell}(q-1)^{\ell}}{(\alpha+1-q ; \alpha ; q)_{\ell}(q ; q)_{\ell} \rho^{\ell}}
\end{aligned}
$$

for $j=0,1,2, \ldots$ We note that, while the roles of $\rho$ and $\alpha / \rho$ are interchangeable when $q>0$, their distinction is essential when $q<0$, as $\rho=\sqrt{-\alpha}$ and $\alpha / \rho=-\sqrt{-\alpha}$.

\section{$5.3 \quad$ Case $\alpha=0, \beta / \sqrt{k} \rightarrow 0$}

With reference to Assumption 3.2, suppose that we are in Theorem 3.5 (ii) with $\rho=0$. Note that $q>0$ in this case, and let

$$
c=\left\lfloor\log _{q} \sqrt{k}\right\rfloor .
$$

Then we have $\sqrt{k} / q^{c} \in[1, q)$. Let $\eta / \sqrt{q-1} \in[1, q]$ be an accumulation point of $\sqrt{k} / q^{c}$, and consider a subnet of $\left(\Gamma_{\lambda}\right)_{\lambda \in \Lambda}$ for which $\sqrt{k} / q^{c} \rightarrow \eta / \sqrt{q-1}$ if $\eta$ is not unique. We note that $k \rightarrow \infty, t \rightarrow 0, c \rightarrow \infty, d-c \rightarrow \infty$, and that

$$
\beta q^{d-2 c} \sim \frac{k(q-1)}{q^{2 c}} \rightarrow \eta^{2}, \quad t \beta \rightarrow 0, \quad t q^{c} \rightarrow \frac{\gamma \sqrt{q-1}}{\eta} .
$$

Using this, (3.4), (5.2), and (5.3), we obtain

$$
\frac{\theta_{c-j}-t k}{\Sigma_{t}(A)} \rightarrow \frac{\eta q^{j}-1 / \eta q^{j}}{\sqrt{q-1}}-\gamma
$$

\footnotetext{
${ }^{6}$ This follows for example from the observation that $\mu((a, b)) \rightarrow \mu_{\infty}((a, b))$ for every bounded open interval $(a, b)$ in $\mathbb{R}$ and [7, Theorem 8.2.17]. When $q>0$, it is also immediate to check that the distribution function of $\mu$ converges to that of $\mu_{\infty}$ at the points of continuity of the latter.
} 


$$
\begin{aligned}
& \frac{m_{c-j}}{|X|} \rightarrow \frac{\left(1+1 / \eta^{2} q^{2 j}\right) q^{-2 j^{2}+j}}{\left(q^{-1} ; q^{-1}\right)_{\infty}\left(-1 / \eta^{2} ; q^{-1}\right)_{\infty}\left(-\eta^{2} / q ; q^{-1}\right)_{\infty} \eta^{4 j}} \\
& \sum_{i=0}^{d} t^{i} v_{i}\left(\theta_{c-j}\right) \rightarrow\left(\gamma \sqrt{q-1} / \eta q^{j+1} ; q^{-1}\right)_{\infty}\left(-\gamma \eta q^{j-1} \sqrt{q-1} ; q^{-1}\right)_{\infty}
\end{aligned}
$$

for $j=0, \pm 1, \pm 2, \ldots$, where we have used

$$
\left(-q^{c-j-d} / \beta ; q\right)_{d+1}=\left(-q^{c-j-d} / \beta ; q\right)_{c+j+1}\left(-q^{2 c-d+1} / \beta ; q\right)_{d-c-j}
$$

for the second formula, and (5.4) for the third one. Again, the measure (5.1) converges weakly to the discrete measure $\mu_{\infty}$ on $\mathbb{R}$ defined by the above limits.

\section{Examples}

There are currently eleven known infinite families of distance-regular graphs having classical parameters with unbounded diameter and such that $q \neq 1$. In this section, we apply the results of the previous sections to these eleven families. For more detailed information on these families, see the references given. It should be remarked that, by virtue of Proposition 3.1, there exist infinitely many choices for the scalar $\gamma$ in Theorem $3.5(i)$ and $(i i)$.

\subsection{Grassmann graphs and twisted Grassmann graphs}

The Grassmann graph $J_{q}(n, d)$ has as vertices the $d$-dimensional subspaces of the $n$-dimensional vector space $\mathbb{F}_{q}^{n}$ over the finite field $\mathbb{F}_{q}$ with $q$ elements, where two vertices $x$ and $y$ are adjacent when $\operatorname{dim} x \cap y=d-1$; cf. [8, Section 9.1]. We always assume that $n \geqslant 2 d$, as $J_{q}(n, d)$ and $J_{q}(n, n-d)$ are isomorphic. The graph $J_{q}(n, d)$ has classical parameters $(d, q, \alpha, \beta)$, where

$$
\alpha=q, \quad \beta=q\left[\begin{array}{c}
n-d \\
1
\end{array}\right] .
$$

Fix $q$ and let $d \rightarrow \infty, t \sqrt{k} \rightarrow \gamma$, and let also $n-2 d+1 \rightarrow 2 \delta$ for some $\delta \in \frac{1}{2} \mathbb{Z}$, so that we have $\beta / \sqrt{k} \rightarrow \rho:=q^{\delta}$. We are in Theorem 3.5 $(i)$, and from the results of Section 5.1 it follows that the measure (5.1) converges weakly to $\mu_{\infty}$, where

$$
\begin{aligned}
\mu_{\infty}( & \left.\frac{q^{\delta+j}+q^{-\delta-j}-q^{\delta}-q^{1-\delta}-\gamma(q-1)}{(q-1) \sqrt{1+\gamma\left(q^{\delta}+q^{1-\delta}\right)}}\right) \\
= & \left(\frac{1}{q^{j(2 \delta+j-1)}}-\frac{1}{q^{(j+1)(2 \delta+j)}}\right)\left(\gamma(q-1) / q^{\delta+j+1} ; q^{-1}\right)_{\infty} \\
& \times{ }_{2} \phi_{1}\left(\begin{array}{c}
q^{-j}, q^{1-2 \delta-j} \\
q
\end{array} \mid q ; \gamma(q-1) q^{\delta+j}\right)
\end{aligned}
$$

for $j=0,1,2, \ldots$ Note that $\delta \geqslant 1 / 2$ and $\alpha / \rho=q^{1-\delta}$, from which it follows that a different choice of $\delta$ gives rise to a different limit in Theorem $3.5(i)$. Hora [16] previously obtained $\mu_{\infty}$ for the vacuum state $\varphi_{0}$, i.e., for $\gamma=0$.

The twisted Grassmann graph $\tilde{J}_{q}(2 d+1, d)$ is defined as follows. Fix a hyperplane $H$ of $\mathbb{F}_{q}^{2 d+1}$. The vertex set consists of the $(d+1)$-dimensional subspaces of $\mathbb{F}_{q}^{2 d+1}$ which are not contained in $H$, together with the $(d-1)$-dimensional subspaces of $H$. Two vertices $x$ and $y$ are adjacent when $\operatorname{dim} x+\operatorname{dim} y-2 \operatorname{dim} x \cap y=2$. See [9]. The graph $\tilde{J}_{q}(2 d+1, d)$ has the same classical parameters as $J_{q}(2 d+1, d)$, and hence we obtain the above measure $\mu_{\infty}$ with $\delta=1$. 


\subsection{Dual polar graphs and Hemmeter graphs}

Consider one of the following vector spaces $V$ endowed with a non-degenerate form:

$C_{d}(q): V=\mathbb{F}_{q}^{2 d}$ with a symplectic form;

$B_{d}(q): V=\mathbb{F}_{q}^{2 d+1}$ with a quadratic form;

$D_{d}(q): V=\mathbb{F}_{q}^{2 d}$ with a quadratic form of Witt index $d$;

${ }^{2} D_{d+1}(q): V=\mathbb{F}_{q}^{2 d+2}$ with a quadratic form of Witt index $d$;

${ }^{2} A_{2 d}(r): V=\mathbb{F}_{q}^{2 d+1}$ with a Hermitian form $\left(q=r^{2}\right)$;

${ }^{2} A_{2 d-1}(r): V=\mathbb{F}_{q}^{2 d}$ with a Hermitian form $\left(q=r^{2}\right)$.

We note that maximal isotropic subspaces of $V$ have dimension $d$. The dual polar graph on $V$ has as vertices these maximal isotropic subspaces, where two vertices $x$ and $y$ are adjacent when $\operatorname{dim} x \cap y=d-1$; cf. [8, Section 9.4]. This graph has classical parameters $\left(d, q, 0, q^{e}\right)$, where we let $e$ be $1,1,0,2,3 / 2,1 / 2$ in the respective types $C_{d}(q), B_{d}(q), D_{d}(q),{ }^{2} D_{d+1}(q),{ }^{2} A_{2 d}(r)$, and ${ }^{2} A_{2 d-1}(r)$. Fix one of these types as well as $q$, and let $d \rightarrow \infty, t \sqrt{k} \rightarrow \gamma$. We have $\beta / \sqrt{k} \rightarrow 0$, and hence we are in Theorem 3.5 (ii) with $\rho=0$. Let the scalar $c$ be as in Section 5.3. Note that

$$
c=\left\lfloor\frac{\log _{q}\left(q^{d}-1\right)-\log _{q}(q-1)+e}{2}\right\rfloor .
$$

Using $d \sim \log _{q}\left(q^{d}-1\right)<d$ and $0 \leqslant \log _{q}(q-1)<1$, we obtain the value of $c$ for sufficiently large $d$ as follows:

\begin{tabular}{c|c|c|c|c|c}
\hline & $e=1$ & $e=0$ & $e=2$ & $e=3 / 2$ & $e=1 / 2$ \\
\hline$d$ even & $d / 2$ & $d / 2-1$ & $d / 2$ & $d / 2$ & $d / 2-1$ \\
$d$ odd & $(d-1) / 2$ & $(d-1) / 2$ & $(d+1) / 2$ & $(d-1) / 2$ & $(d-1) / 2$ \\
\hline
\end{tabular}

For the last two cases ( $e \in\{3 / 2,1 / 2\}$ ), we have also used $q \geqslant 4$ (as $q=r^{2}$ is a square) and $\log _{4} 3=0.792 \ldots$ It follows that $\sqrt{k} / q^{c} \in[1, q)$ has two accumulation points, and considering limits for even $d$ and odd $d$ separately, the scalar $\eta$ from Section 5.3 is given as in the following table:

\begin{tabular}{c|c|c|c|c|c}
\hline & $e=1$ & $e=0$ & $e=2$ & $e=3 / 2$ & $e=1 / 2$ \\
\hline$d$ even & $q^{1 / 2}$ & $q$ & $q$ & $q^{3 / 4}$ & $q^{5 / 4}$ \\
$d$ odd & $q$ & $q^{1 / 2}$ & $q^{1 / 2}$ & $q^{5 / 4}$ & $q^{3 / 4}$ \\
\hline
\end{tabular}

The measure (5.1) converges weakly to the measure $\mu_{\infty}$ as described in Section 5.3. (We do not write down the result here, since there are four values of $\eta$ and also since the substitution of these values does not seem to simplify the formula significantly.)

Note that the graphs $C_{d}(q)$ and $B_{d}(q)$ share the same classical parameters $(d, q, 0, q)$. The extended bipartite double of a graph $\Gamma=(X, R)$ is the graph with vertex set $\mathbb{F}_{2} \times X$, where a vertex $(\epsilon, x)$ is adjacent to $(\epsilon+1, x)$ and all the vertices $(\epsilon+1, y)$ with $x \sim y$. The graph $D_{d}(q)$ is shown to be isomorphic to the extended bipartite double of $B_{d-1}(q)$. The Hemmeter graph $\operatorname{Hem}_{d}(q)$ is then defined as the extended bipartite double of $C_{d-1}(q)$; cf. [8, Section 9.4C]. It has the same classical parameters $(d, q, 0,1)$ as $D_{d}(q)$, so that we obtain the above $\mu_{\infty}$ for $e=0$.

\subsection{Half dual polar graphs and Ustimenko graphs}

Recall that a graph $\Gamma=(X, R)$ is said to be bipartite whenever there is a bipartition $X=$ $X^{+} \sqcup X^{-}$such that no edge is contained in $X^{+}$or $X^{-}$. In this case, a halved graph of $\Gamma$ 
has as vertex set either $X^{+}$or $X^{-}$, where two distinct vertices are adjacent when there is a path of length two joining them in $\Gamma$. The dual polar graph $D_{n}(r)$ and the Hemmeter graph $\operatorname{Hem}_{n}(r)$ are bipartite, and their halved graphs are called the half dual polar graph $D_{n, n}(r)$ and the Ustimenko graph $\mathrm{Ust}_{n}(r)$, respectively; cf. [8, Section 9.4C]. These graphs have classical parameters $(d, q, \alpha, \beta)$, where

$$
d=\left\lfloor\frac{n}{2}\right\rfloor, \quad q=r^{2}, \quad \alpha=r(r+1), \quad \beta=\frac{r\left(r^{m}-1\right)}{r-1}
$$

where $m=2\lceil n / 2\rceil-1$. Fix $r$, and let $d \rightarrow \infty, t \sqrt{k} \rightarrow \gamma$. Note that $\beta / \sqrt{k}$ has two accumulation points $\sqrt{r+1}$ and $r \sqrt{r+1}=\alpha / \sqrt{r+1}$, where $\beta / \sqrt{k} \rightarrow \sqrt{r+1}$ for even $n$, and $\beta / \sqrt{k} \rightarrow$ $r \sqrt{r+1}$ for odd $n$. We consider limits for even $n$ and odd $n$ separately. Set $\epsilon=0$ for even $n$, and $\epsilon=1$ for odd $n$. From the results of Sections 5.1 and 5.2 it follows that the measure (5.1) converges weakly to $\mu_{\infty}$, where

$$
\begin{aligned}
\mu_{\infty}( & \left.\frac{r^{\epsilon+2 j}+r^{-\epsilon-2 j}-r-1-\gamma(r-1) \sqrt{r+1}}{(r-1) \sqrt{r+1+\gamma(r+1)^{5 / 2}}}\right) \\
= & \frac{r^{2 \epsilon+4 j}-1}{r^{\epsilon+2 j}-1} \frac{\left(r^{-1} ; r^{-2}\right)_{\infty}}{r^{(\epsilon+j)(2 j+1)}\left(r^{-2} ; r^{-2}\right)_{\infty}}\left(\gamma(r-1) \sqrt{r+1} / r^{\epsilon+2 j+2} ; r^{-2}\right)_{\infty} \\
& \times{ }_{2} \phi_{1}\left(\begin{array}{c}
r^{-2 j}, r^{1-2 \epsilon-2 j} \mid \\
r
\end{array} \mid r^{2} ; \gamma r^{\epsilon+2 j}(r-1) \sqrt{r+1}\right)
\end{aligned}
$$

for $j=0,1,2, \ldots$, where we understand that $\left(r^{0}-1\right) /\left(r^{0}-1\right)=1$ when $\epsilon=j=0$.

\subsection{Second classical parameters for dual polar graphs ${ }^{2} A_{2 d-1}(r)$}

The Hermitian dual polar graph ${ }^{2} A_{2 d-1}(r)$ has another set of classical parameters $(d, q, \alpha, \beta)$, where (cf. (3.7))

$$
q=-r, \quad \alpha=\frac{r(r+1)}{1-r}, \quad \beta=\frac{r\left(1+(-r)^{d}\right)}{1-r} .
$$

Fix $r$, and let $d \rightarrow \infty, t \sqrt{k} \rightarrow \gamma$. Note that $\beta / \sqrt{k}$ has two accumulation points $\pm \sqrt{-\alpha}$, where $\beta / \sqrt{k} \rightarrow-\sqrt{-\alpha}$ for even $d$, and $\beta / \sqrt{k} \rightarrow \sqrt{-\alpha}$ for odd $d$. We consider limits for even $d$ and odd $d$ separately. According to whether $\beta / \sqrt{k} \rightarrow \pm \sqrt{-\alpha}$, the measure (5.1) converges weakly to $\mu_{\infty}$, where

$$
\begin{aligned}
\mu_{\infty}( & \left.\frac{\mp \sqrt{r}(-r)^{j} \pm \sqrt{r}^{-1}(-r)^{-j}}{\sqrt{r^{2}-1}}-\gamma\right) \\
& =\frac{\left(r^{2 j+1}+1\right)\left(r^{-1} ;-r^{-1}\right)_{\infty}}{r^{(j+1)^{2}}\left(-r^{-1} ;-r^{-1}\right)_{\infty}}\left(\mp \gamma(-r)^{-j-1} \sqrt{\frac{r^{2}-1}{r}} ;-r^{-1}\right)_{\infty}\left(\mp \gamma(-r)^{j-1} \sqrt{\frac{r^{2}-1}{r}} ; r^{-2}\right)_{j}
\end{aligned}
$$

for $j=0,1,2, \ldots$ Here we have again used (5.4) to get the result. We may routinely verify that this measure is identical to the one in Section 6.2 with $e=1 / 2$, using

$$
\left(r^{-1} ;-r^{-1}\right)_{\infty}\left(-r^{-1} ; r^{-2}\right)_{\infty}=1
$$

which is a special case of Lebesgue's identity; see, e.g., [11]. 


\subsection{Sesquilinear forms graphs}

There are four infinite families of sesquilinear forms graphs, all of which are Cayley graphs.

The bilinear forms graph $\operatorname{Bil}(d \times e, q)$ has as vertices the $d \times e$ matrices over $\mathbb{F}_{q}$, where two vertices $x$ and $y$ are adjacent when $\operatorname{rank}(x-y)=1$; cf. [8, Section 9.5A]. We always assume that $d \leqslant e$, as $\operatorname{Bil}(d \times e, q)$ and $\operatorname{Bil}(e \times d, q)$ are isomorphic. The graph $\operatorname{Bil}(d \times e, q)$ has classical parameters $\left(d, q, q-1, q^{e}-1\right)$. Fix $q$ and let $d \rightarrow \infty, t \sqrt{k} \rightarrow \gamma$, and $e-d \rightarrow 2 \delta$ for some $\delta \in \frac{1}{2} \mathbb{Z}$, so that $\beta / \sqrt{k} \rightarrow \rho:=q^{\delta} \sqrt{q-1}$. The measure (5.1) converges weakly to $\mu_{\infty}$, where

$$
\begin{aligned}
& \mu_{\infty}\left(\frac{q^{\delta+j}-q^{\delta}-q^{-\delta}-\gamma \sqrt{q-1}}{\sqrt{q-1+\gamma\left(q^{\delta}+q^{-\delta}\right)(q-1)^{3 / 2}}}\right) \\
& \quad=\frac{(-1)^{j}\left(q^{-2 \delta-j-1} ; q^{-1}\right)_{\infty}}{(q ; q)_{j} q^{2 \delta j+\left(\begin{array}{l}
j \\
2
\end{array}\right)}\left(\gamma \sqrt{q-1} / q^{\delta+j+1} ; q^{-1}\right)_{\infty^{2}} \phi_{0}\left(\begin{array}{c}
q^{-j}, q^{-2 \delta-j} \\
-
\end{array} \mid q ; \gamma q^{\delta+j} \sqrt{q-1}\right)}
\end{aligned}
$$

for $j=0,1,2, \ldots$ Since $\delta \geqslant 0$ and $\alpha / \rho=q^{-\delta} \sqrt{q-1}$, it follows that a different choice of $\delta$ gives rise to a different limit in Theorem $3.5(i)$.

The alternating forms graph $\operatorname{Alt}(n, r)$ has as vertices the $n \times n$ skew-symmetric matrices with zero diagonal over $\mathbb{F}_{r}$, where two vertices $x$ and $y$ are adjacent when $\operatorname{rank}(x-y)=2$; cf. [8, Section 9.5B]. It has classical parameters $(d, q, \alpha, \beta)$, where

$$
d=\left\lfloor\frac{n}{2}\right\rfloor, \quad q=r^{2}, \quad \alpha=r^{2}-1, \quad \beta=r^{m}-1
$$

where $m=2\lceil n / 2\rceil-1$. Fix $r$, and let $d \rightarrow \infty, t \sqrt{k} \rightarrow \gamma$. Note that we can apply the above computation with $e:=m / 2$. Hence we consider limits for even $n$ and odd $n$ separately, and set $\delta=-1 / 4$ for even $n$ and $\delta=1 / 4$ for odd $n$. We then obtain the above measure $\mu_{\infty}$ with $q=r^{2}$.

The quadratic forms graph Qua $(n-1, r)$ has as vertices the quadratic forms on $\mathbb{F}_{r}^{n-1}$, where two vertices $x$ and $y$ are adjacent when $\operatorname{rank}(x-y) \in\{1,2\}$. See [8, Section 9.6] for a precise description. This graph has the same classical parameters as $\operatorname{Alt}(n, r)$, and thus the result is the same as above.

Finally, the Hermitian forms graph $\operatorname{Her}\left(d, r^{2}\right)$ has as vertices the $d \times d$ Hermitian matrices over $\mathbb{F}_{r^{2}}$, where two vertices $x$ and $y$ are adjacent when $\operatorname{rank}(x-y)=1 ; \mathrm{cf}$. [8, Section 9.5C]. It has classical parameters $\left(d,-r,-r-1,-(-r)^{d}-1\right)$. Fix $r$, and let $d \rightarrow \infty, t \sqrt{k} \rightarrow \gamma$. We consider limits for even $d$ and odd $d$ separately, where $\beta / \sqrt{k} \rightarrow-\sqrt{r+1}$ for even $d$, and $\beta / \sqrt{k} \rightarrow \sqrt{r+1}$ for odd $d$. According to whether $\beta / \sqrt{k} \rightarrow \pm \sqrt{r+1}$, the measure (5.1) converges weakly to $\mu_{\infty}$, where

$$
\begin{aligned}
\mu_{\infty}\left(\mp \frac{(-r)^{j}}{\sqrt{r+1}}-\gamma\right)= & \frac{\left(-(-r)^{-j-1} ;-r^{-1}\right)_{\infty}}{(-r ;-r)_{j}(-r)^{\left(\begin{array}{l}
j \\
2
\end{array}\right)}\left(\mp \gamma \sqrt{r+1} /(-r)^{j+1} ;-r^{-1}\right)_{\infty}} \\
& \times{ }_{2} \phi_{0}\left(\begin{array}{c}
(-r)^{-j},-(-r)^{-j} \\
-
\end{array} \mid-r ; \pm \gamma(-r)^{j} \sqrt{r+1}\right)
\end{aligned}
$$

for $j=0,1,2, \ldots$.

Remark 6.1. Set $\gamma=0$ in the above examples, which is the case when we take scaling limits of the vacuum state $\varphi_{0}$. The measure $\mu_{\infty}$ is then an affine transformation of the discrete orthogonality measure of the Al-Salam-Chihara polynomials (cf. [25, Section 3.8]) for Sections 6.1 and 6.3, that of the continuous $q^{-1}$-Hermite polynomials (cf. [25, Section 3.26]) for Sections 6.2 and 6.4, and that of the Al-Salam-Carlitz II polynomials (cf. [25, Section 3.25]) with base $q^{-1}$ for Section 6.5. For the discrete orthogonality measures of the first two families of orthogonal polynomials, see, e.g., [2, equation (3.82)], [3, equation (3.18)], and [21, equation (6.31)]. 


\section{Acknowledgements}

The authors thank the anonymous referees for valuable comments. HT thanks Professor Tom Koornwinder for letting him know that the measure $\mu_{\infty}$ with $\gamma=0$ in Section 6.1 corresponds to the Al-Salam-Chihara polynomials, and for providing relevant references. Part of this work was done while MK was visiting Tohoku University from February to July 2020, supported by K.N. Toosi University of Technology, Office of Vice-Chancellor for Global Strategies and International Affairs. NO and HT were supported by JSPS KAKENHI Grant Number JP19H01789. HT was also supported by JSPS KAKENHI Grant Numbers JP17K05156 and JP20K03551. This work was also partially supported by the Research Institute for Mathematical Sciences at Kyoto University.

\section{References}

[1] Accardi L., Lu Y.G., Volovich I., Quantum theory and its stochastic limit, Springer-Verlag, Berlin, 2002.

[2] Askey R., Ismail M., Recurrence relations, continued fractions, and orthogonal polynomials, Mem. Amer. Math. Soc. 49 (1984), iv+108 pages.

[3] Atakishiyev N.M., Klimyk U., Duality of $q$-polynomials, orthogonal on countable sets of points, Electron. Trans. Numer. Anal. 24 (2006), 108-180, arXiv:math.CA/0411249.

[4] Bang S., Dubickas A., Koolen J.H., Moulton V., There are only finitely many distance-regular graphs of fixed valency greater than two, Adv. Math. 269 (2015), 1-55, arXiv:0909.5253.

[5] Bannai E., Ito T., Algebraic combinatorics. I. Association schemes, The Benjamin/Cummings Publishing Co., Inc., Menlo Park, CA, 1984.

[6] Biggs N., Algebraic graph theory, 2nd ed., Cambridge Mathematical Library, Cambridge University Press, Cambridge, 1993.

[7] Bogachev V.I., Measure theory, Vols. I, II, Springer-Verlag, Berlin, 2007.

[8] Brouwer A.E., Cohen A.M., Neumaier A., Distance-regular graphs, Ergebnisse der Mathematik und ihrer Grenzgebiete (3), Vol. 18, Springer-Verlag, Berlin, 1989.

[9] Van Dam E.R., Koolen J.H., A new family of distance-regular graphs with unbounded diameter, Invent. Math. 162 (2005), 189-193.

[10] Van Dam E.R., Koolen J.H., Tanaka H., Distance-regular graphs, Electron. J. Combin. (2016), \#DS22, 156 pages, arXiv:1410.6294.

[11] Fu A.M., A combinatorial proof of the Lebesgue identity, Discrete Math. 308 (2008), 2611-2613.

[12] Gudder S.P., Quantum probability, Probability and Mathematical Statistics, Academic Press, Inc., Boston, MA, 1988.

[13] Hashimoto Y., Quantum decomposition in discrete groups and interacting Fock spaces, Infin. Dimens. Anal. Quantum Probab. Relat. Top. 4 (2001), 277-287.

[14] Hashimoto Y., Hora A., Obata N., Central limit theorems for large graphs: method of quantum decomposition, J. Math. Phys. 44 (2003), 71-88.

[15] Hashimoto Y., Obata N., Tabei N., A quantum aspect of asymptotic spectral analysis of large Hamming graphs, in Quantum Information, III (Nagoya, 2000), Editors T. Hida, K. Saitô, World Sci. Publ., River Edge, NJ, 2001, 45-57.

[16] Hora A., Central limit theorems and asymptotic spectral analysis on large graphs, Infin. Dimens. Anal. Quantum Probab. Relat. Top. 1 (1998), 221-246.

[17] Hora A., Gibbs state on a distance-regular graph and its application to a scaling limit of the spectral distributions of discrete Laplacians, Probab. Theory Related Fields 118 (2000), 115-130.

[18] Hora A., Asymptotic spectral analysis on the Johnson graphs in infinite degree and zero temperature limit, Interdiscip. Inform. Sci. 10 (2004), 1-10.

[19] Hora A., Obata N., Quantum probability and spectral analysis of graphs, Theoretical and Mathematical Physics, Springer, Berlin, 2007.

[20] Hora A., Obata N., Asymptotic spectral analysis of growing regular graphs, Trans. Amer. Math. Soc. 360 (2008), 899-923. 
[21] Ismail M.E.H., Masson D.R., $q$-Hermite polynomials, biorthogonal rational functions, and $q$-beta integrals, Trans. Amer. Math. Soc. 346 (1994), 63-116.

[22] Ivanov A.A., Shpectorov S.V., The association schemes of dual polar spaces of type ${ }^{2} A_{2 d-1}\left(p^{f}\right)$ are characterized by their parameters if $d \geq 3$, Linear Algebra Appl. 114/115 (1989), 133-139.

[23] Kelley J.L., General topology, Graduate Texts in Mathematics, Vol. 27, Springer-Verlag, New York - Berlin, 1975.

[24] Koekoek R., Lesky P.A., Swarttouw R.F., Hypergeometric orthogonal polynomials and their $q$-analogues, Springer Monographs in Mathematics, Springer-Verlag, Berlin, 2010.

[25] Koekoek R., Swarttouw R.F., The Askey scheme of hypergeometric orthogonal polynomials and its $q$-analog, Report 98-17, Delft University of Technology, 1998, available at http://aw.twi.tudelft.nl/ koekoek/ askey $\cdot$ html.

[26] Leonard D.A., Orthogonal polynomials, duality and association schemes, SIAM J. Math. Anal. 13 (1982), 656-663.

[27] Meyer P.-A., Quantum probability for probabilists, Lecture Notes in Math., Vol. 1538, Springer-Verlag, Berlin, 1993.

[28] Nica A., Speicher R., Lectures on the combinatorics of free probability, London Mathematical Society Lecture Note Series, Vol. 335, Cambridge University Press, Cambridge, 2006.

[29] Obata N., Spectral analysis of growing graphs. A quantum probability point of view, SpringerBriefs in Mathematical Physics, Vol. 20, Springer, Singapore, 2017.

[30] Pan Y.-J., Weng C.-W., A note on triangle-free distance-regular graphs with $a_{2} \neq 0$, J. Combin. Theory Ser. B 99 (2009), 266-270.

[31] Parthasarathy K.R., An introduction to quantum stochastic calculus, Modern Birkhäuser Classics, Birkhäuser/Springer Basel AG, Basel, 1992.

[32] Tanaka H., Vertex subsets with minimal width and dual width in $Q$-polynomial distance-regular graphs, Electron. J. Combin. 18 (2011), \#P167, 32 pages, arXiv:1011.2000.

[33] Terwilliger P., Q-polynomial distance-regular graphs containing a singular line with cardinality at least 3 , unpublished manuscript.

[34] Terwilliger P., The subconstituent algebra of an association scheme. I, J. Algebraic Combin. 1 (1992), 363388.

[35] Terwilliger P., The subconstituent algebra of an association scheme. II, J. Algebraic Combin. 2 (1993), 73-103.

[36] Terwilliger P., The subconstituent algebra of an association scheme. III, J. Algebraic Combin. 2 (1993), $177-210$.

[37] Terwilliger P., Kite-free distance-regular graphs, European J. Combin. 16 (1995), 405-414.

[38] Terwilliger P., Two linear transformations each tridiagonal with respect to an eigenbasis of the other, Linear Algebra Appl. 330 (2001), 149-203, arXiv:math.RA/0406555.

[39] Terwilliger P., Leonard pairs and the q-Racah polynomials, Linear Algebra Appl. 387 (2004), 235-276, arXiv:math.QA/0306301.

[40] Terwilliger P., Two linear transformations each tridiagonal with respect to an eigenbasis of the other; comments on the parameter array, Des. Codes Cryptogr. 34 (2005), 307-332, arXiv:math.RA/0306291.

[41] Terwilliger P., Žitnik A., The quantum adjacency algebra and subconstituent algebra of a graph, J. Combin. Theory Ser. A 166 (2019), 297-314, arXiv:1710.06011.

[42] Voiculescu D.V., Dykema K.J., Nica A., Free random variables, CRM Monograph Series, Vol. 1, Amer. Math. Soc., Providence, RI, 1992.

[43] Weng C.-W., D-bounded distance-regular graphs, European J. Combin. 18 (1997), 211-229.

[44] Weng C.-W., Classical distance-regular graphs of negative type, J. Combin. Theory Ser. B 76 (1999), 93-116. 